\title{
On Designing Practical Long Range Near Infrared-based Face Recognition Systems
}

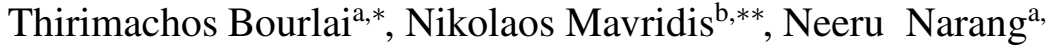 \\ ${ }^{a}$ West Virginia University \\ PO Box 6201, Morgantown, West Virginia 26506 \\ ${ }^{b}$ IRML, NCSR Demokritos \\ GR-15310, Aghia Paraskevi, Attiki, Greece
}

\begin{abstract}
Although automated face recognition (AFR) is a well-studied problem with a history of more than three decades, it is still far from being considered a solved problem for the case of difficult exposure conditions, such as during night-time, in environments with unconstrained lighting, or at large distances from the camera. However, in practical forensic scenarios, it is often the case that investigators operate in difficult conditions, where cross-session data need to be matched and where, grouping of the data in the context of demographic information (constitute the grouping in terms of gender, ethnicity) may be used in order to assist law enforcement officials, forensic investigators and security personnel in human identification practices. In this paper, we discuss the challenges in designing a practical near infrared (NIR) FR system and, more specifically, study the problems of intraspectral, cross-spectral, i.e. VIS-NIR, intra-distance and cross-distance NIR FR, in indoors, outdoors, day-time and night-time environments. Furthermore, we
\end{abstract}

\footnotetext{
*Principal corresponding author

** Corresponding author

Email address: Thirimachos. Bourlai@mail.wvu. edu (Thirimachos Bourlai)
} 
propose the usage of a multi-feature scenario dependent fusion scheme that can enhance recognition performance. We also investigate which scenarios used, related to datasets, features useful for face matching or their combination, are most beneficial to the identification accuracy of NIR FR systems, when the gallery set is composed of either visible or NIR band face images. Thus, we illustrate that the selection of specific feature extraction techniques and their fusion are often the key design aspects that can turn practically non-functional systems to effective systems with real-world applicability. As a result, such a strategy can significantly extend the range of conditions under which automated NIR FR systems can operate.

Keywords: Forensics, Face Recognition, Near Infrared, Cross-Scenario Face Matching, Demographic Information, Night-time, Long-Distance, Score Level Fusion.

\section{Introduction}

2 Face recognition systems have long been a very important tool for security 3 applications. Databases of passport-style photos of millions of people have tradi4 tionally been part of the citizen records of most nations. Such photos are usually standoff distance (i.e. the distance between the camera (source) and the face (target)), as well as the background, for example, are kept within tightly controlled limits. However, for both security-related as well as consumer-based applications of face recognition, the conditions of the materials to be used for training or testing a human recognition system are much more varied. The variability of illumination sources and background, as well as poses variation and even partial facial 
occlusions, are usually the norm rather than the exception in freely-taken material, such as surveillance videos, photos posted on the internet etc. Furthermore, forensic material often needs to be taken in even harsher conditions: for example, during night-time, or at moderately long distances from the subject, often surpassing thirty meters (or about 100 feet) standoff distance.

Under such conditions, traditional face recognition approaches, such as those based on Local Binary Patterns [1] or Linear Discriminant Analysis [2], often provide unsatisfactory results, which can be practically unusable in a court of law, where typically face-based evidence needs to be extremely accurate so that it can provide to law enforcement officers a solid lead, to either identify or rule out suspects. Thus, it is a fact that multiple directions toward improving the design of more efficient and practical FR systems are being explored.

This work is an effort to investigate some of the challenges of unconstrained face recognition by focusing on three main directions (see Fig. 1): (i) the usage of both visible and near infrared sensors, (ii) dealing with challenging matching scenarios, where the problem domain is composed of: homogeneous or intra-spectral, and heterogeneous or cross-spectral matching studies, while also the face images are acquired using different cameras, at day-time or night-time conditions, indoors or outdoors, at different standoff distances (up to more than 100 meters away) and (iii) the usage of a multi-feature scenario depended fusion (MFSDF) face matching scheme (our proposed approach), with and without the support of grouping in the context of demographic information, which can assist in improving FR performance at the aforementioned challenging matching conditions.

One example pertaining to the first two directions, deals with the problem of identifying people both at day-time and night-time environments, at variable dis- 


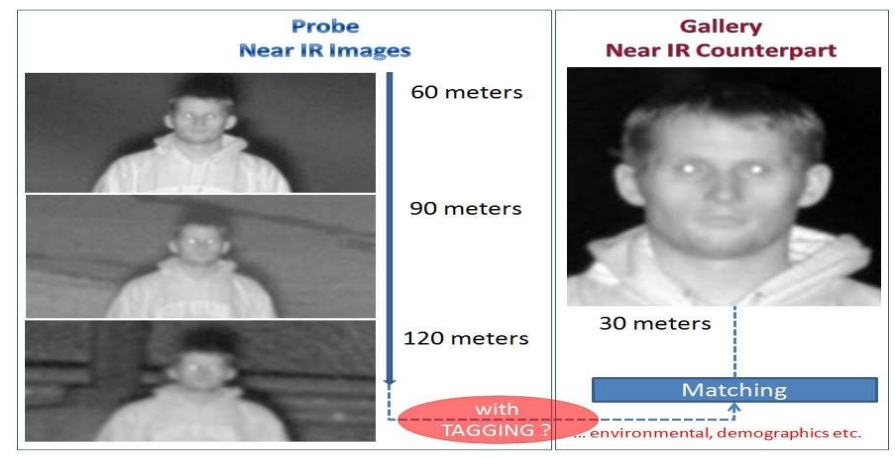

Figure 1: Problem overview: matching cross-scenario face data, in which the probe face images are captured in the near infrared band when operating under difficult conditions, is a very challenging task. In this study, we, first, investigate variable face matching scenarios using academic, commercial and our proposed system. In addition, we performed a study where the expectation is to improve FR performance by utilizing male and female groups in the context of demographic information of data in face matching studies.

tances and illumination conditions. This can be accomplished by using various types of visible (e.g. with telephoto lenses) and infrared sensors (Near, ShortWave, Mid-Wave, and Long-Wave IR), under either single-spectral or multi-spectral recognition settings $[3,4]$. We also know that there are certain settings and configurations that provide increased identification performance during night-time operations, when using passive IR sensors [3], active IR sensors [5] or visible band sensors that operate during day-time and at medium distances [6].

One example pertaining to the third direction, deals with the problem of improving identification performance using our proposed multi-feature (LBP, Gabor and HOG) scenario-dependent fusion scheme. Our proposed scheme is tested on two different dual-band face datasets collected as part of two separate studies $[7,8]$. This is beneficial for the purpose of this study, since the subjects on each dataset are different and the faces of the subjects were captured under similar 
conditions (i.e. in terms of standoff distance and overall environment) but using different sensors (details will be provided in section 3.2).

The usage of male and female groups in the context of demographic information of data is also considered. Filtering is performed before face matching for the purpose of improving face recognition performance. A practical example where one of the state-of-the-art FR commercial software was assisted by demographic data to work efficiently is discussed in [9]. This is the case of the April's 2013 bomb attacks in Boston, U.S.A. where two men, shown in pixelated photos and video footage, were suspected of involvement in the event. These suspects were successfully face-based identified (as part of a proof of concept study after the event), only when demographic filtering was used (e.g. age range and gender), as a prior step to FR matching, i.e. a filtered search was performed where each probe was only compared against gallery images with similar demographic data.

While all aforementioned directions are necessary when designing a FR system, there are other problems that FR operators need to deal with. In practical applications, one of the problems with human face-based recognition is that it is often the case where the training material (i.e. the gallery dataset of face images, normally captured in a controlled environment of a set of individuals enrolled in the dataset) is only available in the visible spectrum (see Fig. 2). Thus, one needs to perform the very challenging task of matching cross-scenario face images. One way of attempting to deal with such a challenge is through photometric normalization [10], utilizing algorithms such as contrast limited adaptive histogram equalization (CLAHE) [11]. While such algorithms often are shown to improve identification performance, their capability in practical applications is limited due to the fact that face images of suspects (probes), available from surveillance data, 

images (gallery), typically captured in the visible band.

\section{Controlled, Cooperative User Scenarios (No Tagging)}
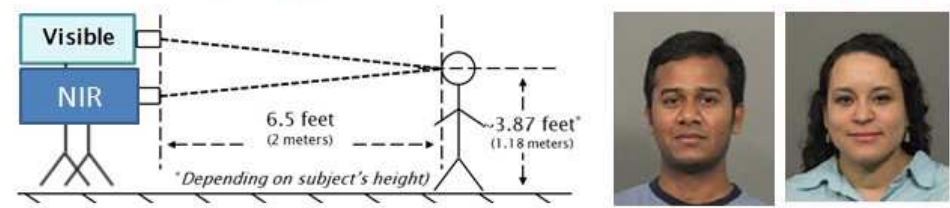

Long Range, Uncontrolled User Scenarios (No Tagging)

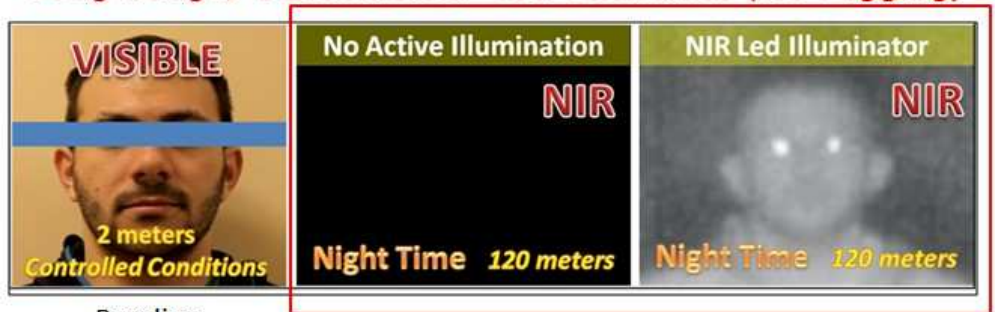

Baseline

Figure 2: Data collection scenarios of both visible and infrared face data, under controlled (top image) or uncontrolled (bottom image) conditions. The questions that need to be answered are (i) 'what is the baseline FR performance when performing cross-scenario matching?'and, (ii) 'can FR benefit from context of demographic information of data for the face images collected by surveillance cameras?'

In this paper, motivated by the above state of affairs, we are presenting highly promising concrete quantitative results arising from the design and evaluation of a NIR FR system (in comparison with a conventional visible FR system) that operates at night-time and at long distances (both baseline and cross-scenario studies have been performed). In this paper we also present results arising from before (baseline) and after the usage of our proposed fusion approach, when (i) we used 
either the same or different datasets for training and testing. All NIR face images were collected outdoors at night time conditions, while the visible counterparts of the NIR face images were collected either indoors or outdoors, (ii) we extended the training set with more subjects and images, (iii) we deal with only male or female subsets for performance evaluation. Hence, the overview of our work is to study what are the effects of matching cross-scenario face data (collected at different conditions; using different sensors) have on the identification performance of a NIR FR system, while we also investigate which conditions and scenarios, associated with each face image captured, can further enhance recognition performance.

For the rest of the paper, we will start by providing an overview of related existing background research, and then, describe our system, our proposed methodological approach and the results obtained. Next, we will provide a discussion, including interesting observations as well as future directions, before proceeding to a concise conclusion of our findings, which illustrates the benefits of our proposed matching scheme and usage of female and male groups in the context of demographic information of data towards improving FR performance.

\section{Background}

Automated Face Recognition is a field with considerable history, with major publications having already started in the 80s. A somewhat outdated but quite complete review of the field can be found in [5]. Classic methods include Eigenfaces [12] as well as variants [13], Elastic Graph Matching [14], Linear Discriminant Analysis [15], Kernel-Based methods, Hidden Markov Models [16], all the way to more recent methods using sparse representations [17] and ensembles of 
classifiers [18]. Apart from academic systems, a number of high-performance commercially available systems also exist, such as FaceVACS [19], PittPatt [20], Identity Tools G8 [21] and NeoFace by NEC (www.nec.com).

Regarding face recognition in difficult conditions, such as night-time or large distances, infrared and multi-spectral imaging has provided promising results. In [22], the Long-Distance Heterogeneous Face (LDHF) Database is presented, which contains face images collected in an outdoor environment at distances of 1 meters, 60 meters, 100 meters, and 150 meters, with both visible light (VIS) face images captured in daytime and NIR face images captured in nighttime. Results for intra-spectral as well as cross-spectral face matching are provided, and a FR algorithm using SIFT and random subspaces is presented. However, the 100 meter and 150 meter ranges have quite poor results. In [23] a SWIR face recognition system is discussed that was used from the collection of multi-spectral data. Cross-scenario matching experiments are discussed in [4]. There, both commercial as well as academic FR algorithms are used, including LBP [1] and LTP [24]. Also, a number of photometric normalization techniques are utilized, e.g. CLAHE [11], single scale retinex with logarithmic (SSRlog) or with arc-tangent transformation (SSRatan), in order to facilitate intra-spectral and cross-spectral matching. Furthermore, datasets on SWIR and MWIR bands were collected, at multiple progressive difficulty levels. Promising results regarding the utility of other FR cross-scenarios, e.g. in the MWIR band is provided at [3].

When focusing only in NIR FR approaches published in the literature, we can state that they can be grouped into the following main categories: (i) NIR FR: Both gallery and probe sets are composed of only NIR images [25, 26, 27, 28, 29 , 30, 31]. (ii) Image Synthesis: usage of image synthesis to convert NIR images to 
Table 1: NIR FR literature, including the FR matching category, as well as the approaches, environment and standoff distances used (see also table at [8]).

\begin{tabular}{|c|c|c|c|c|}
\hline Category & Literature & Approach & Indoors/Outdoors & Standoff Distances \\
\hline \multirow{4}{*}{$\begin{array}{c}\text { Homogeneous FR } \\
\text { NIR vs. NIR }\end{array}$} & Li et al., 2007 [29] & LBP, Adaboost & Indoors & $0.5 \sim 1 \mathrm{~m}$ \\
\cline { 2 - 5 } & Zhang et al., 2010 [28] & Gabor, DBC & Indoors & $0.8 \sim 1.2 \mathrm{~m}$ \\
\cline { 2 - 5 } & Huang et al. 2007 [26] & ELBP, Adaboost & Indoors & $\sim 1.5 \mathrm{~m}$ \\
\cline { 2 - 5 } & Shen et al. 2012 [31] & DBC, Adaboost & Indoors & $\sim 1.5 \mathrm{~m}$ \\
\cline { 2 - 5 } & Kang et al. [8] & Filtering, SIFT, MLBP & Indoors/Outdoors & $1 \mathrm{~m}, 60 \mathrm{~m}, 100 \mathrm{~m}, 150 \mathrm{~m}$ \\
\cline { 2 - 5 } & Bourlai et al. [7] & CSU Face Matchers & Indoors/Outdoors & $1.5 \mathrm{~m}, 30 \mathrm{~m}, 60 \mathrm{~m}, 90 \mathrm{~m}, 120 \mathrm{~m}$ \\
\hline \multirow{4}{*}{$\begin{array}{c}\text { Heterogeneous FR } \\
\text { VIS-NIR }\end{array}$} & Yi et al. 2007 [38] & PCA-CCA & Indoors & $\sim 1.5 \mathrm{~m}$ \\
\cline { 2 - 5 } & Liao et al. 2009 [39] & DoG, MB-LBP,G. Adaboost & Indoors & $\sim 1.5 \mathrm{~m}$ \\
\cline { 2 - 5 } & Maeng et al. 2011 [22] & DoG-SIFT & Indoors & $1 \mathrm{~m}, 60 \mathrm{~m}, 100 \mathrm{~m}, 150 \mathrm{~m}$ \\
\cline { 2 - 5 } & Klare \& Jain 2013 [40] & MLBP, SIFT,LDA, Kernel & Indoors & $0.8 \sim 1.2 \mathrm{~m}$ \\
\cline { 2 - 5 } & Kang et al. [8] & Filtering, SIFT, MLBP & Indoors/Outdoors & $1 \mathrm{~m}, 60 \mathrm{~m}, 100 \mathrm{~m}, 150 \mathrm{~m}$ \\
\cline { 2 - 5 } & Bourlai et al. [7] & CSU Face Matchers & Indoors/Outdoors & $1.5 \mathrm{~m}, 30 \mathrm{~m}, 60 \mathrm{~m}, 90 \mathrm{~m}, 120 \mathrm{~m}$ \\
\hline
\end{tabular}

visible ones, before FR experiments are conducted [32, 33, 34], and (iii) Crossscenario FR: the probe face images are NIR, while the gallery face image are visible (typically mug shots collected indoors and at a fixed standoff distance, a case that is more realistic in a law enforcement scenario). In [7] (part of this study is discussed in this paper), a night-time, middle-range near infrared sensor is evaluated at four distances ranging from 30 to 120 meters, and results on both intra-spectral (visible to NIR) as well as intra-distance matching are provided. Thus, what we propose is an approach related to the first but mainly to the third category. Table 1 summarizes representative NIR FR approaches reported in the literature pertaining to the first and last category.

Examples of context-assisted FR include [35, 36], where sensed and inferred contextual meta-data are utilized in order to enhance recognition in camera-phone photos, as well as [37], where the interplay between people, events, and locations is utilized in order to create a unified recognition system. 
From all the aforementioned published work in NIR FR, the closest paper to ours is [8]. The main differences are the following: (1) we used a NIR camera sensor with a NIR illuminator, while in [8] they used a visible camera with telephoto lenses and a NIR illuminator. The developed system from [8], can capture better quality images at longer distances than our developed system. (2) The demographic data, time and period of collection are different. (3) A MFSDF face matching scheme is proposed that significantly improves the performance of face recognition system from the database collected under cross-distance and crossspectral scenarios. (4) In our paper, we exploited further the cross-scenarios with the context of demographic information (gender class) and showed it is useful to improve face identification performance. (5) The database has video sequences of full frontal mid-range NIR and visible 232 face images of each subject, resulting in a total of $103 \times 5$ videos $(103 \times 4$ NIR 233 outdoors and 103 visible indoors $)$ per subject. To the best of our knowledge, our study is the first in the literature where the NIR face images were captured using a NIR camera system with a NIR illuminator, outdoors, only at night-time (pitch black), at variable distances, and, the demographic information is also captured and used for identification in this database.

What follows, is a description of baseline experiments, our proposed methodological approach, including the NIR camera system used for the purpose of this study, the face image data collected. Then, we will discuss the FR algorithms tested for our same- and cross-scenario matching and we will proceed with the description of our new proposed method and the analysis that we carried out towards answering our main research questions as discussed in Section 1 and Fig. 2. 

a precision pan-tilt platform (see Fig. 3).
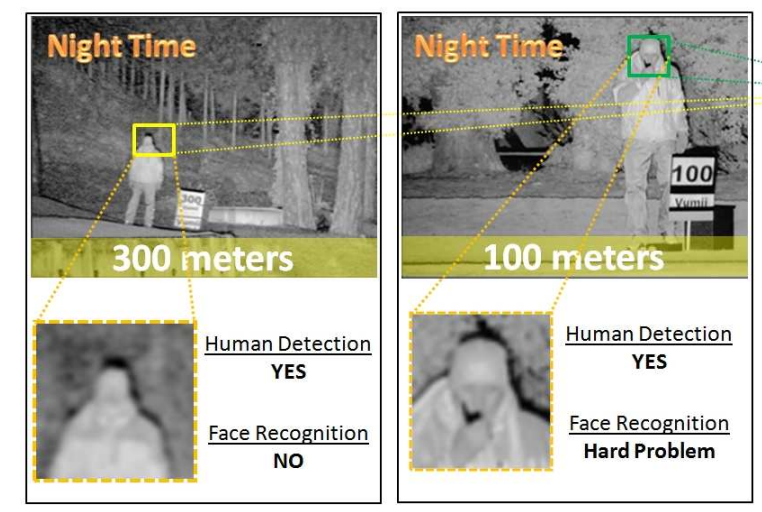

\section{Methodological Approach}

\subsection{Night-Time Imagery}

Figure 3: Overview of the NIR camera system with the capability to acquire face images day and night at mid-ranges, i.e., from about 70 and up to 400 feet (20 - 120 meters). We can see the challenges with night-time FR at mid-ranges: without active illumination FR is not possible, while with active illumination FR is possible but a very challenging problem, especially at standoff distances further than 60 meters.

The mid-range camera system used in this work is a Near-IR camera (provided by Vumii Imaging Inc.) that operates at $850 \mathrm{~nm}$. It is a unique outdoor perimeter and border surveillance camera system, and its bulk version integrates (i) a NIR LED illuminator that is invisible to the human eye, (ii) a camera with high-magnification optics (continuous optical long-focal length zoom) placed on

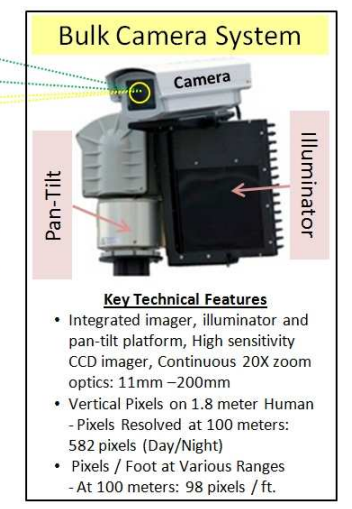

\subsection{Live Subject-Capture Setup and Datasets}

Two cameras were used in our live subject capture setup: (a) Canon EOS 5D Mark II, i.e. a digital SLR camera that has a 21.1-mega-pixel full-frame CMOS sensor, and (b) a NIR mid-range imaging system that leverages a focused-beam 

period of 20 days. Then, the subjects' mug shots was also taken using the visible
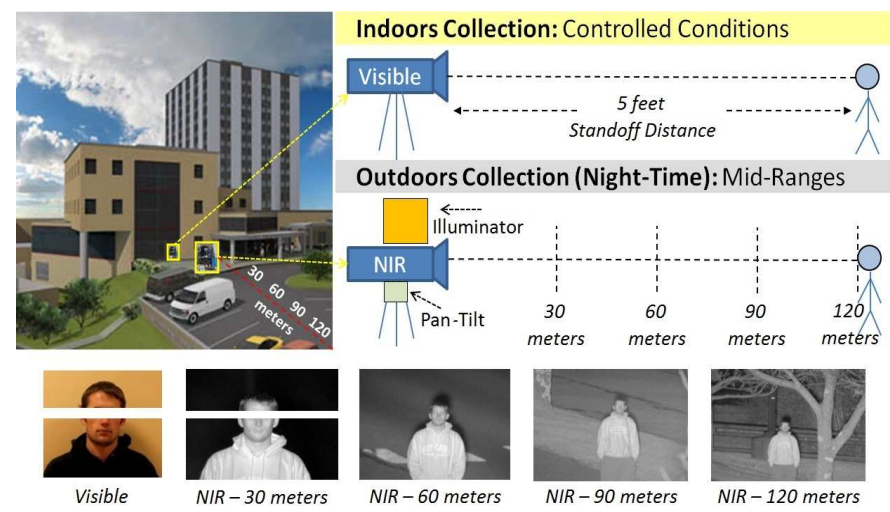

array LED technology combined with an optimized imager, optics, and pan-tilt platform. The camera also has an IR illumination source focused LED beam array $(850 \mathrm{~nm})$. The live face capture configuration we used is illustrated in Fig. 4.

The NIR database was assembled outdoors at night time spanning over a time

Figure 4: The live subject-capture setup using the visible and mid-range NIR cameras. At the bottom of the figure we can see face image samples acquired by our system.

the bottom of the figure we can see face image samples acquired by our system.

camera in an indoors controlled environment. In total, 103 subjects (69 male + 34 female) participated in this experiment. The more detail information about the data preparation for FR studies is provided in [7]. ${ }^{1}$

Table 1 provides information about relevant work to ours, while Table 2 below compares the WVU, NIR Mid-Range and LDHF face databases for cross-distance and cross-spectral face matching. The pre-processing steps and the FR studies we performed will be described below, starting first, with our proposed multi-feature

\footnotetext{
${ }^{1}$ Data Release of the (WVU) NIR Mid-Range Face Dataset: This is, currently, a private database with availability determined on a case-by-case basis. If interested in working with this database, please contact Thirimachos Bourlai, at ThBourlai@mail.wvu.edu.
} 
Table 2: Summary of face image databases for cross-spectral and cross-distance matching using long range data. Note: WVU database consists of video sequences for each subject. Still images were selected from those sequences to perform the FR studies. In this work $103 \times 8$ NIR outdoors (4 for gallery and 4 for probes) and 103 visible indoors face images per subject were used.

\begin{tabular}{|c|c|c|c|c|c|c|}
\hline Database & \# Subjects & \# Images & Sensors & Day/Night & Indoors/Outdoors & Distances \\
\hline \multirow{2}{*}{ LDHF[8] } & 100 & 200 & Visible & Day & Indoors & $1 \mathrm{~m}$ \\
\cline { 2 - 7 } & 100 & 600 & Telephoto, NIR illuminator & Day and Night & Outdoors & $1 \mathrm{~m}, 60 \mathrm{~m}, 100 \mathrm{~m}, 150 \mathrm{~m}$ \\
\hline \multirow{2}{*}{ NIR WVU[7] } & 103 & $\mathrm{n} \times 103$ & Visible & Night & Indoors (mugshots) & $1.5 \mathrm{~m}$ \\
\cline { 2 - 7 } & 103 & $\mathrm{n} \times 103$ & NIR System & Night & Outdoors & $30 \mathrm{~m}, 60 \mathrm{~m}, 90 \mathrm{~m}, 120 \mathrm{~m}$ \\
\hline
\end{tabular}

scenario dependent fusion scheme.

\subsection{Multi-Feature Scenario Dependent Fusion}

To perform cross-scenarios face matching, we propose a multi-feature scenario dependent fusion (MFSDF) scheme. It involves four main steps: (i) Preprocessing of images with and without the usage of mask, (ii) Feature extraction using global and local descriptors, such as Gabor, HOG and LBP, (iii) Learning Subspace (iv) Fusion of scores based on a decision level scheme.

Pre-processing: Geometric normalization of images is performed to compensate for slight perturbations in the frontal pose. We adopt two different methods: with and without the usage of a mask.

Normalization With Mask: To perform the normalization with mask, we use the technique developed from AFIES (more detail information is provided in [41]). A sequence of normalization steps are performed including, integer to float conversion, geometric normalization (lines up human chosen eye coordinates), masking (crops the image using an elliptical mask and image borders such that only the face from forehead to chin and cheek to cheek is visible), and pixel normalization 


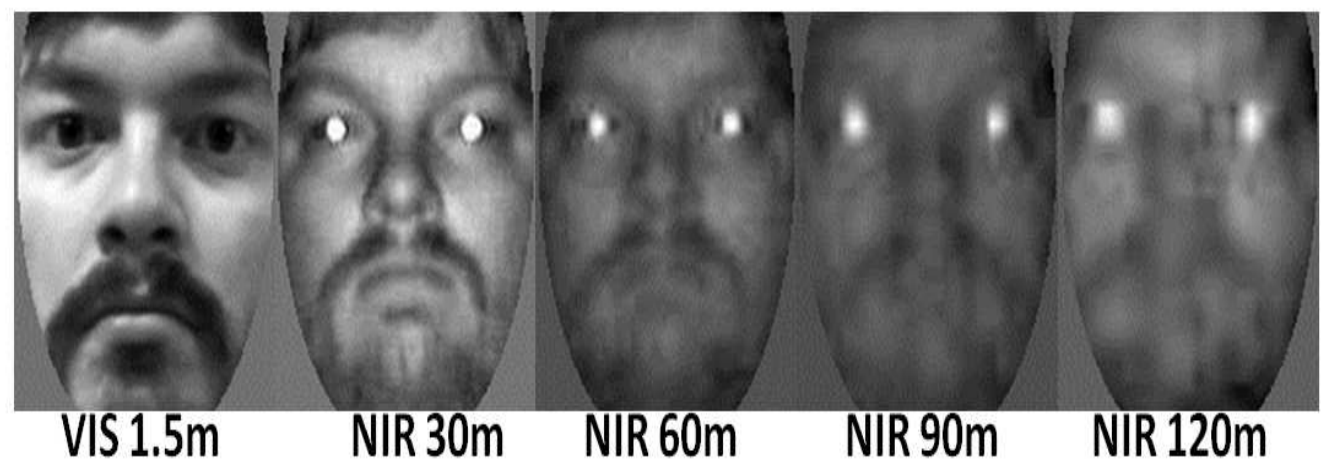

Figure 5: Normalized elliptical face mask with a standard size of $130 \times 150$ pixels.

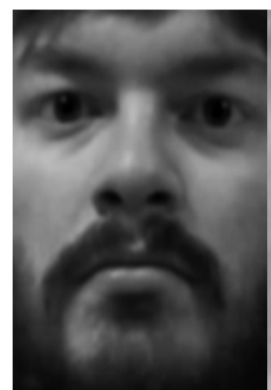

\section{VIS $1.5 \mathrm{~m}$}

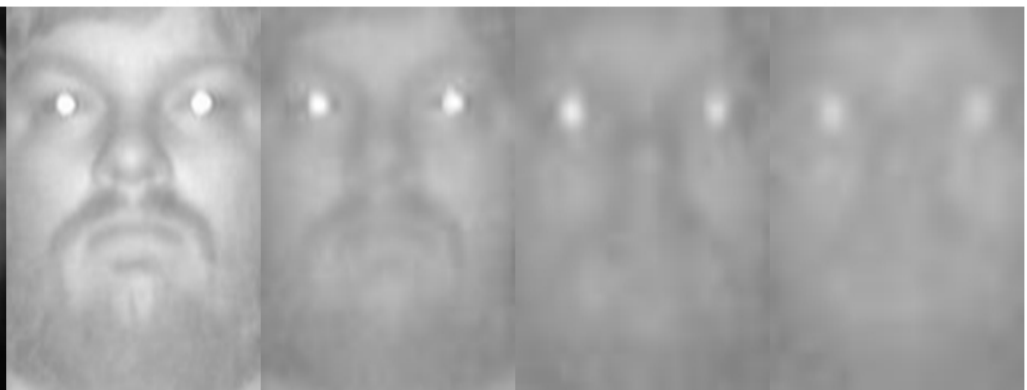

NIR 30m NIR 60m NIR 90m NIR $120 \mathrm{~m}$

Figure 6: Normalized face region with a standard size of $128 \times 128$ pixels.

217

218

cally normalize the images, based on the located eye coordinates, the canonical 
faces are automatically generated by applying an affine transformation. Faces are first aligned by placing the coordinates of the eyes in the same row, such that the slope between the right and left eye is zero degrees. Finally, cropped the face region to a standard size of $128 \times 128$ pixels (see Fig. 6).

Feature Extraction: (i) LBP: provides texture-based information. It is highly discriminative, efficient and proved to performs well when used as part of a FR system [24]. It performs well even when the background is not uniform and edges are noisy. (ii) Gabor: features are used to extract face shapes and appearance. The Gabor wavelets transformation provides desirable features and captures properties like spatial locality, spatial frequency and orientation selectivity. The features extracted from Gabor filtered images are more robust to illumination and facial expressions. In this work, we used 40 Gabor wavelets with the scale value $\nu$ lies in range from $\{0,1,2,3,4\}$ and eight orientations $\mu$ in the range of $\{0,1, \ldots, 7\}$. (iii) HOG: This is one of the most well-known local shape descriptors in computer vision [40]. The main idea is to extract the local orientation information rather than the magnitude of image patches. Images are divided into overlapping or non-overlapping cells and neighboring cells consist of a local region that represented as a block. The local shape information (HOG features) is computed for each block. The extracted features of blocks are concatenated into a vector $x$ (components of $x$ are local features of image). We selected the cell size $8 \times 8$ and block size $2 \times 2$.

Feature Subspace: A set of features is based on LBP, Gabor and HOG methods for NIR and VIS images. To reduce the dimensionality of the feature space, we adopted the method used by Tan et al. [24], where they only used LBP and Gabor image feature descriptors. In our work we extended the feature space to LBP, 
Gabor and HOG and, further than that, used the KLDA learning based analysis method to reduce the dimensionality problem.

Fusion of scores: It is based on decision level scenarios. We select 11 different scenarios: (i) the first three scenarios are based on the usage of single feature descriptor, namely the Gabor, HOG or LBP, (ii) the next six scenarios are based on the usage of two features descriptors, using sum and weighted fusion schemes. In the sum fusion scheme, we select three combinations: Gabor+LBP, Gabor+HOG and HOG+LBP. In the weighted fusion scheme, we select combinations with two features and, then, weights are assigned based on the performance of the individual feature descriptor. (iii) The last two scenarios are based on all three feature descriptors, using the sum (Gabor+LBP+HOG) and weighted fusion scheme where weights are assigned to each descriptor based on the performance scores (distance scores). Finally, we performed an empirical evaluation in order to select the scenario which provides us with best rank-1 scores.

\subsection{Baseline Face Recognition Matchers}

In our cross-scenario FR studies we used both commercial and academic FR software. While the pre-processing routines employed by the commercial software are not known, the academic software employed the following pre-processing and face matching modules.

- Pre-Processing: This step is the same as the pre-processing step in Section 3.3. - Face Recognition Tools: Both commercial and academic software were employed to perform the FR experiments. In terms of the commercial off-the-shelf (COTS) software, we used the Identity Tools G8 (www.11id.com) provided by L1 Systems. In the academic software we used standard FR methods are provided by the Academic Face Identification Evaluation System (AFIES) CSU [41], in- 
cluding Principle Components Analysis (PCA) [42, 43, 44], a combined Principle Components Analysis and Linear Discriminant Analysis algorithm (PCA+LDA) [45], and the Bayesian Intra-personal/Extra-personal Classifier (BIC) using either the Maximum likelihood (ML) or the Maximum a posteriori (MAP) hypothesis [46].

\section{Face Recognition Studies}

\subsection{Matching Across Different Scenarios}

We evaluate system performance using the CSU, the commercial and our proposed FR methods, and utilize the datasets briefly discussed in subsection 3.2. To check the efficiency of our system, we selected 5 different sets, with different training and testing combinations and an extended gallery dataset. We performed the following experiments:

Experiment 1: Visible vs. Visible (intra-spectral) face matching - it provides the rank-1 identification rates of a conventional FR system. For that purpose we use only a commercial software.

Experiment 2: NIR vs. NIR (intra-spectral) and intra-distance face matching at all distances available. The experiments performed were $30 \mathrm{~m} v \mathrm{~s} .30 \mathrm{~m}$ (baseline, best quality images for the camera sensor used), $60 \mathrm{~m} v \mathrm{~s} .60 \mathrm{~m}, 90 \mathrm{~m} \mathrm{vs}$. $90 \mathrm{~m}$, and $120 m$ vs. $120 m$.

Experiment 3: We also match cross-spectral and cross-distance images, i.e. the baseline visible images to NIR images captured at 30m, 60m, 90m and $120 \mathrm{~m}$ respectively.

Experiment 4: We compare cross-distance images, i.e. the baseline NIR images $(30 m)$ to NIR images captured at $60 \mathrm{~m}$, $90 \mathrm{~m}$ and $120 \mathrm{~m}$ respectively. Equal impor- 
tantly, as a case study (see more information below) we also investigated whether demographic information can assist in improving cross-distance and cross-spectral FR performance.

Experiment 5: We extended the gallery data, using LDHF database collected from [8]. For training based FR matchers (AFIES and proposed method), used either the same or different datasets for training and testing. We extended the training set with more subjects and images for the database collected under different environment conditions i.e. indoor or outdoor, different distances. We used variable training sets but due to the page limitation of this paper we cannot include and discuss all results.

While the main cross-scenario FR experiments (1-2) described above (Section 4.1) are easy to understand, we provide more discussion on experiments 3,4 and 5 . Here we investigate whether, the change in distance $(30 \mathrm{~m}$ to $60 \mathrm{~m}, 90 \mathrm{~m}$ and $120 \mathrm{~m})$ and sensor (gallery in VIS and Probe in NIR), affects the performance of face recognition system.

We also propose the MFSDF face matching scheme to improve the identification rank-1 performance of the system, for the database collected under un-controlled conditions. We also investigate whether demographic information, in particular the gender class (male and female) and extended gallery data, contribute to FR performance under certain conditions, and this has never been investigated in the past, as we have commented above.

Please note that the identification performance of the system is evaluated through the cumulative match characteristic (CMC) curve. The CMC curve measures the $1: m$ identification system performance, and judges the ranking capability of the identification system. 


\section{Experimental Results}

What follows is the description of two sets of FR experiments using a set of commercial and academic face matching algorithms. In the first set of experiments, no grouping in the context of demographic information is used. Our experiments aim to illustrate how our baseline NIR FR system operates when compared to a conventional visible FR system (where face images are captured under controlled conditions). We need to know what is the rank-1 identification rate when matching NIR to NIR good quality, long range face images (30m vs. $30 \mathrm{~m}$ ). Finally, we need to know the extent of which the rank-1 identification rate is affected when standoff distance increases. This is established by performing intra- and cross-distance NIR FR matching when going from 30 meters and up to 120 meters away. Cross-spectral and cross-distance matching is also investigated using the same commercial, academic and proposed algorithms. In the second set of experiments, i.e. Experiment as discussed in Section 4.1, we are trying to determine whether the usage grouping in the context of demographic information can enhance recognition performance. Hence, we investigate which combinations of available data are most beneficial to the identification accuracy of the NIR FR system.

Table 3, depicts information about the total number of, datasets, cross-scenarios and face recognition algorithms are selected. In this work, we have selected the datasets with and without the context of demographic information. For without the context of demographic information, all data is used and with demographic information gender class (male and female group) is used. For each dataset, we have randomly divided the $50 \%$ data as training set and rest of data set is used as testing set. There is no overlap between the training and testing images. In each 
Table 3: Summary of total number of face recognition experiments are performed for each dataset, with and without grouping in the context of demographic information. To perform the FR experiments, two FR cross-scenarios are selected cross-distance (CD) and cross-spectral (CS).

\begin{tabular}{|c|c|c|c|c|}
\hline Datasets & $\begin{array}{c}\text { Train/Test } \\
\text { Datasets }\end{array}$ & \# Scenarios & $\begin{array}{c}\text { AFIES, COTS and } \\
\text { Proposed MFSDF }\end{array}$ & $\begin{array}{c}\text { \# Total } \\
\text { Experiments }\end{array}$ \\
\hline All & 4 & 3 CD + 4 CS & $6+1+2$ & 252 \\
\hline Male & 4 & 3 CD + 4 CS & $6+1+2$ & 252 \\
\hline Female & 4 & 3 CD + 4 CS & $6+1+2$ & 252 \\
\hline
\end{tabular}

experiment, the subjects that used in training, were not used for the testing. This process is repeated several different times, using random selection of training and testing set each time. Two cross-scenarios are selected: CD and CS. For CD NIR vs. NIR face matching is performed for 3 sets: $30 \mathrm{~m} v s .60 \mathrm{~m}, 30 \mathrm{~m} v \mathrm{~s} .90 \mathrm{~m}$ and $30 m$ vs. $120 m$. For CS VIS vs. NIR face matching is performed for 4 sets: $1.5 m$ vs. $30 \mathrm{~m}, 1.5 \mathrm{~m}$ vs. $60 \mathrm{~m}, 1.5 \mathrm{~m}$ vs. $90 \mathrm{~m}$ and $1.5 \mathrm{~m}$ vs. $120 \mathrm{~m}$. Cross-scenarios are investigated using academic, commercial (non-training based) and the proposed MFSDF face matching scheme. In table 3 we can see the total number of face matching experiments performed for each dataset.

\subsection{Baseline and Matching Results Before Grouping in the Context of Demo-} graphic Information

Inter-Distance Matching: In this, we compare NIR to NIR, intra-distance face images. The experiments performed were $30 \mathrm{~m} v \mathrm{~s}$. $30 \mathrm{~m}$ (baseline, best quality images), $60 m$ vs. $60 m$, $90 m$ vs. $90 m$, and $120 m$ vs. $120 m$. For this purpose we employed G8 (COTS) and the academic software (AFIES) [41]) while the training set size was varied to determine whether larger training sets will result in higher 
Table 4: In this table we investigate the intra-spectral, intra-distance matching scenarios without demographic information: experimental results when running all CSU FR algorithms when using $20 \%, 40 \%$ and $60 \%$ of the NIR data for training and the rest data $(80 \%, 60 \%$ and $40 \%$ respectively) for testing. When CSU was used, the experiments were run 10 times and the rank-1 scores presented here are the means. G8 was also tested for each scenario. Note that there is one case (90m) where rank-1 was about $38 \%$ vs. $59 \%$ with the $120 \mathrm{~m}$ data. This was because the experiment was run once and we noticed that some $90 \mathrm{~m}$ face images selected for matching were more heavily affected by background noise (e.g. passing cars and illumination) due to the time of the collection.

\begin{tabular}{|c|c|c|c|c|c|c|c|c|c|c|c|c|}
\hline \multirow{3}{*}{ FR Algorithm } & \multicolumn{12}{|c|}{ Intra-Spectral (NIR vs. NIR), Intra-Distance Matching (Rank-1) } \\
\hline & \multicolumn{3}{|c|}{$30 \mathrm{~m}$} & \multicolumn{3}{|c|}{$60 \mathrm{~m}$} & \multicolumn{3}{|c|}{$90 \mathrm{~m}$} & \multicolumn{3}{|c|}{$120 \mathrm{~m}$} \\
\hline & $20 \%$ & $40 \%$ & $60 \%$ & $20 \%$ & $40 \%$ & $60 \%$ & $20 \%$ & $40 \%$ & $60 \%$ & $20 \%$ & $40 \%$ & $60 \%$ \\
\hline Bayesian MAP & 0.9898 & 0.9879 & 0.9952 & 0.9042 & 0.9486 & 0.9491 & 0.9546 & 0.9778 & 0.9962 & 0.9240 & 0.9617 & 0.9815 \\
\hline Bayesian ML & 0.9950 & 0.9976 & 0.9979 & 0.9943 & 0.9958 & 0.9973 & 0.9467 & 0.9675 & 0.9833 & 0.9164 & 0.9524 & 0.9975 \\
\hline LDA Euclidean & 0.9923 & 0.9994 & 1.000 & 0.9905 & 0.9986 & 1.000 & 0.9617 & 0.9911 & 1.000 & 0.9229 & 0.9798 & 1.000 \\
\hline LDA Ida_Soft & 0.9922 & 0.9994 & 1.000 & 0.9892 & 0.9984 & 1.000 & 0.9598 & 0.9907 & 1.000 & 0.9200 & 0.9817 & 1.000 \\
\hline PCA Euclidean & 0.9794 & 0.9873 & 1.000 & 0.9639 & 0.9008 & 0.9872 & 0.9107 & 0.9313 & 0.9476 & 0.8782 & 0.9137 & 0.9405 \\
\hline G8 & \multicolumn{3}{|c|}{0.9564} & \multicolumn{3}{|c|}{0.6942} & \multicolumn{3}{|c|}{0.3738} & \multicolumn{3}{|c|}{0.5923} \\
\hline
\end{tabular}

recognition performance independent of whether we are operating at 30 meters or 120 meters standoff distance. Hence, (a) we randomly selected 20, 40, 60\% of the images for training and the rest images were used for testing, and (b) this process was repeated 10 times. The identification performance results of intra-spectral experiments are summarized in Table 4.

Based on the results of the baseline experiments, we determined that the results from AFIES outperformed the COTS software, where the identification rate is $37 \%$ for $90 m$ and $59 \%$ for $120 m$ (see Table 4).

Cross-Distance Matching: In this experiment, we compared cross-distance face images, i.e. the baseline NIR face images $(30 \mathrm{~m})$ were compared to NIR images captured at $60 \mathrm{~m}, 90 \mathrm{~m}$ and $120 \mathrm{~m}$ respectively. The intra-spectral, cross-distance experiments are summarized in Table 5, Fig. 7 (NIR 30m vs. NIR 60m) and Fig. 8 
Table 5: In this table we investigate the intra-spectral, cross-distance matching scenarios (without demographic filtering): experimental results when running all FR algorithms using $50 \%$ of the NIR data for training and the rest of the data for testing. For each algorithm including, AFIES, COTS and proposed MFSDF face matching scheme, the experiments were run several times and the rank-1 scores presented here are the means.

\begin{tabular}{|c|c|c|c|}
\hline \multirow[b]{2}{*}{ FR Algorithm } & \multicolumn{3}{|c|}{ Intra-Spectral (NIR vs. NIR), Cross-Distance Matching (Rank-1) } \\
\hline & $\begin{array}{c}30 \text { vs. } 60 \mathrm{~m} \\
50 \%\end{array}$ & $\begin{array}{c}30 \text { vs. } 90 \mathrm{~m} \\
50 \%\end{array}$ & $\begin{array}{c}30 \text { vs. } 120 \mathrm{~m} \\
50 \%\end{array}$ \\
\hline AFIES & & & \\
\hline Bayesian MAP & 0.85 & 0.52 & 0.36 \\
\hline Bayesian ML & 0.86 & 0.60 & 0.38 \\
\hline LDA Euclidean & 0.88 & 0.61 & 0.38 \\
\hline LDA lda_Soft & 0.88 & 0.64 & 0.40 \\
\hline PCA Euclidean & 0.68 & 0.27 & 0.20 \\
\hline PCA MahaCosine & 0.86 & 0.68 & 0.46 \\
\hline COTS & 0.16 & 0.02 & 0.01 \\
\hline Proposed Methods & & & \\
\hline MFSDF Without Mask & 0.89 & 0.66 & 0.55 \\
\hline MFSDF With Mask & 0.90 & 0.68 & 0.54 \\
\hline Fusion of MFSDF (best set) & & & \\
\hline With and Without Mask & 0.93 & 0.81 & 0.64 \\
\hline
\end{tabular}

(NIR $30 \mathrm{~m} v s$. NIR $90 \mathrm{~m}$ and NIR $30 \mathrm{~m}$ vs. NIR 120m).

Based on the results, we determined that proposed method and AFIES achieves similar identification results for NIR $30 \mathrm{~m}$ gallery matched against NIR $60 \mathrm{~m}$ probe. The rank-1 score is $88 \%$ for AFIES (LDA-EUC and LDA lda-soft) and $93 \%$ from the proposed method (fusion of mask and non-mask). On the other hand, identification results are very low using COTS software, where the rank-1 score accuracy is $16 \%$. For both $90 \mathrm{~m}$ and $120 \mathrm{~m}$ NIR probe images, proposed approach achieves significant improvement compared to AFIES. For example for 90m rank-1 score is 


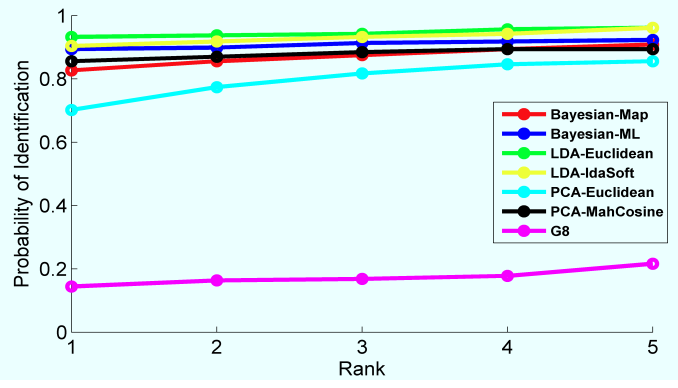

(a) AFIES and COTS

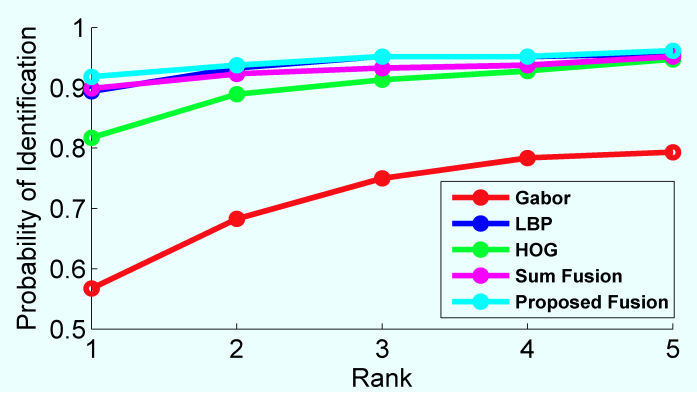

(c) Proposed MFSDF applied to normalized face images without mask

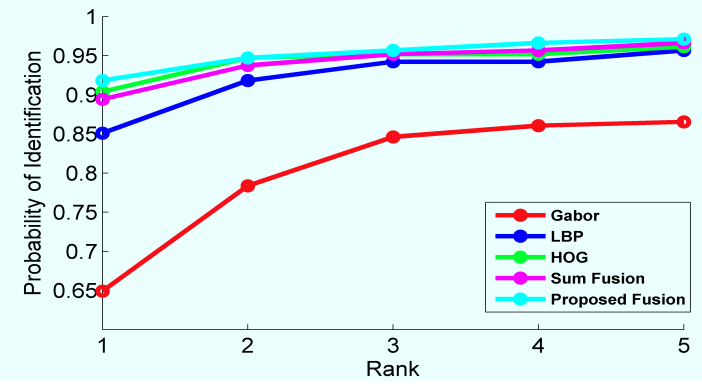

(b) Proposed MFSDF applied to normalized face masks

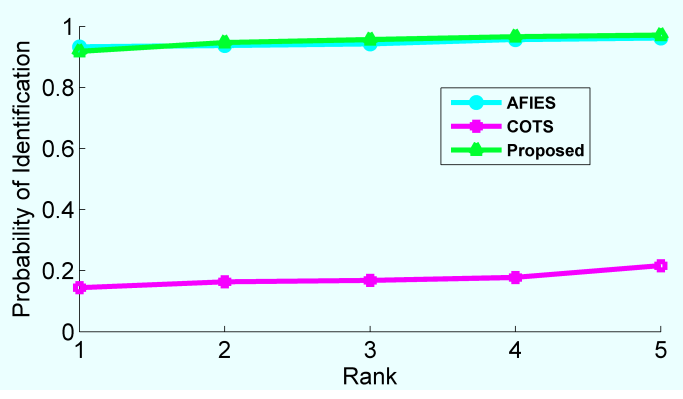

(d) AFIES and COTS vs. Proposed

Figure 7: Intra-spectral, cross-distance matching scenarios for NIR 30m (Gallery) against NIR 60m (Probe): Performance of baseline face recognition and proposed MFSDF face matching scheme for cross-distance images (without demographic information). Each algorithm was run several times and here rank scores are presented from best performing set.

$81 \%$ when using our proposed method, when compared to only $68 \%$ from AFIES (PCA MahaCosine). For 120m, AFIES achieves $46 \%$ identification rate, while the proposed method achieves $64 \%$ rank-1 accuracy.

The CMC curves for the first 5 ranks, for the best performed set out of randomly selected sets, are represented in Figs. 7 and 8 . The best rank-1 scores 


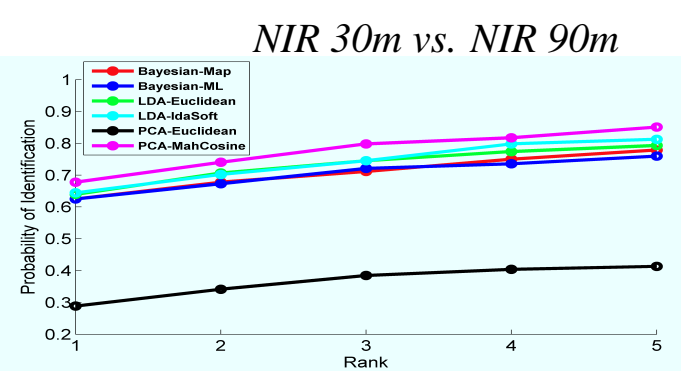

(a) AFIES

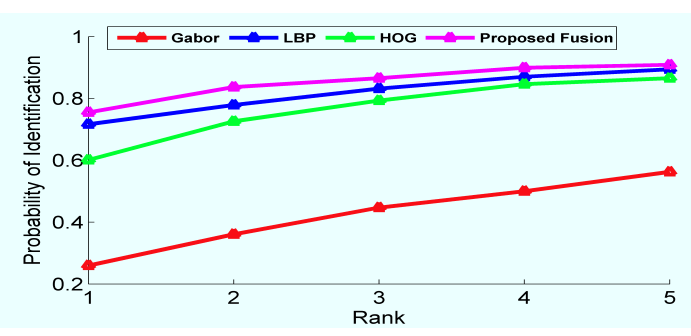

(c) Proposed With Mask

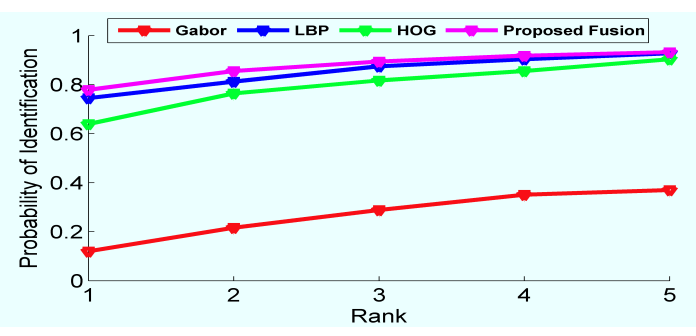

(e) Proposed Without Mask

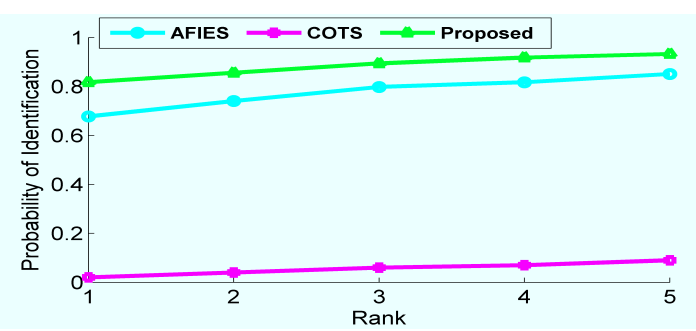

(g) AFIES and COTS vs. Proposed

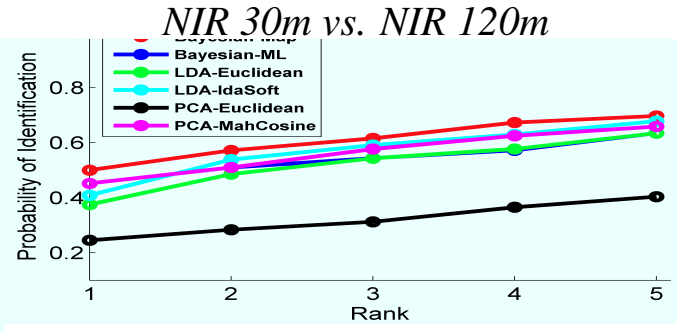

(b) AFIES

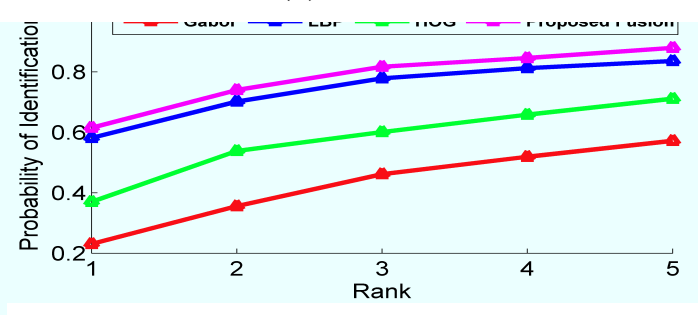

(d) Proposed With Mask

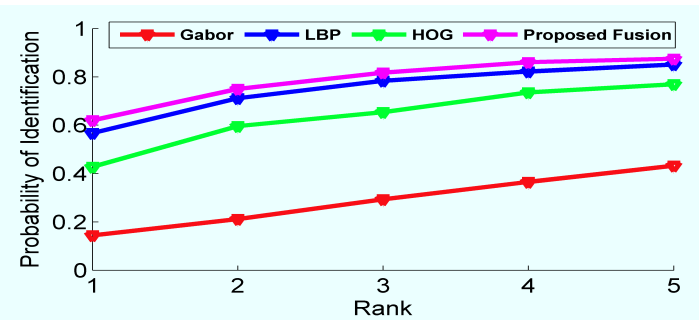

(f) Proposed Without Mask

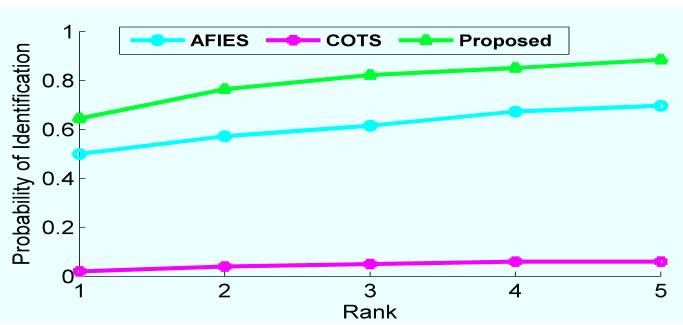

(h) AFIES and COTS vs. Proposed

Figure 8: Intra-spectral, cross-distance matching scenarios for NIR 30m against NIR 90m (Left) and NIR 30m against NIR 120m (Right) (without grouping in the context of demographic information): Each algorithm was run several times and here the rank scores are presented from best set. 
Table 6: In this table we investigate the cross-spectral, cross-distance matching scenarios (without grouping in context of demographic information): experimental results when using $50 \%$ of the data for training and the rest for testing. Experiments were run several times and rank-1 scores presented here are the means.

\begin{tabular}{|c|c|c|c|c|}
\hline & \multicolumn{3}{|c|}{ Intra-Spectral (NIR vs. NIR), Cross-Distance Matching (Rank-1) } \\
Gallery: VIS 1.5m vs. & NIR 30 m & NIR 60 m & NIR 90 m & NIR 120 m \\
FR Algorithms & $\mathbf{5 0 \%}$ & $\mathbf{5 0 \%}$ & $\mathbf{5 0 \%}$ & $\mathbf{5 0 \%}$ \\
\hline \hline AFIES & & & & 0.15 \\
Bayesian MAP & 0.19 & 0.15 & 0.21 & 0.13 \\
Bayesian ML & 0.18 & 0.16 & 0.23 & 0.11 \\
LDA Euclidean & 0.19 & 0.20 & 0.13 & 0.12 \\
LDA lda_Soft & 0.19 & 0.22 & 0.13 & 0.08 \\
PCA Euclidean & 0.08 & 0.08 & 0.07 & 0.01 \\
PCA MahaCosine & 0.12 & 0.10 & 0.09 & 0.01 \\
\hline \hline COTS & 0.48 & 0.03 & 0.02 & 0.16 \\
\hline \hline Proposed Methods & & & & 0.16 \\
MFSDF Without Mask & 0.56 & 0.34 & 0.21 & \\
MFSDF With Mask & 0.50 & 0.37 & 0.23 & \\
\hline \hline
\end{tabular}

achieved using our method are 77\% (no mask), 75\% (with mask) and $81 \%$ for fusion of mask and no mask for the $90 \mathrm{~m}$ (see Fig. 8) and $64 \%$ for fusion of mask and no mask method for $120 m$ probe.

Cross-Spectral Matching: We compared visible to NIR face images captured at $30 \mathrm{~m}, 60 \mathrm{~m}, 90 \mathrm{~m}$ and $120 \mathrm{~m}$. For cross-spectral, experiments are summarized in Table 6 and Fig. 9 (VIS vs. NIR $30 \mathrm{~m}$ and VIS vs. NIR 60m). In all tables we can see the mean rank-1 identification rates when using AFIES, COTS and proposed MFSDF system.

Based on the results, we determined that proposed method achieves better 
VIS $1.5 m$ vs. NIR $30 m$

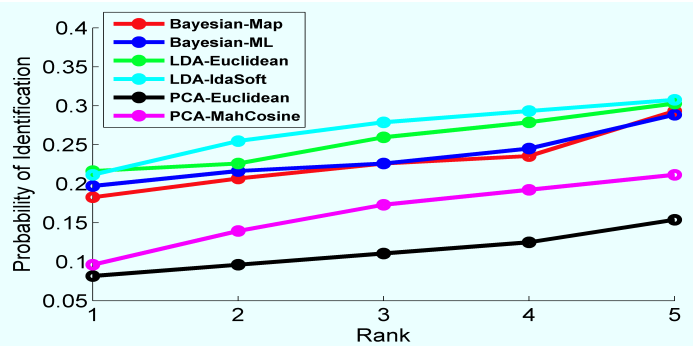

(a) AFIES (VIS vs. NIR 30m)

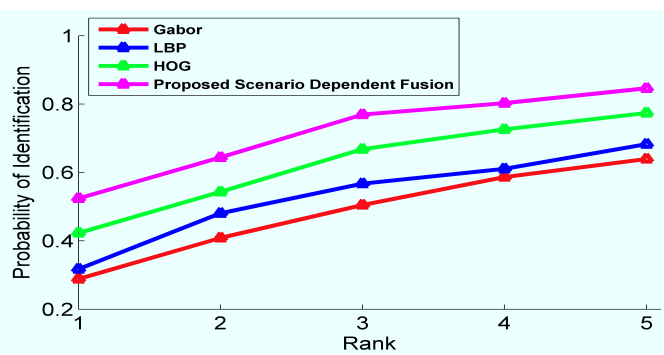

(c) Proposed With Mask

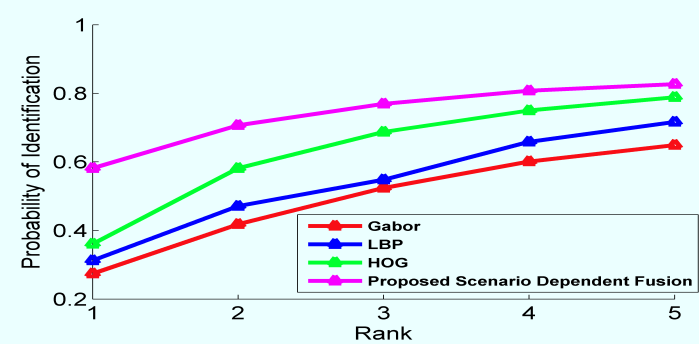

(e) Proposed Without Mask

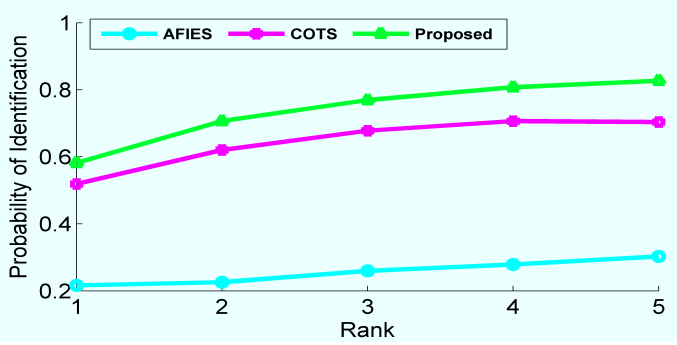

(g) AFIES and COTS vs. Proposed

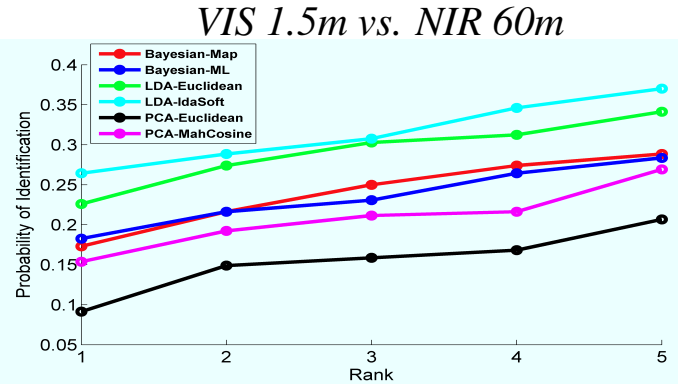

(b) AFIES (VIS vs. NIR 60m)

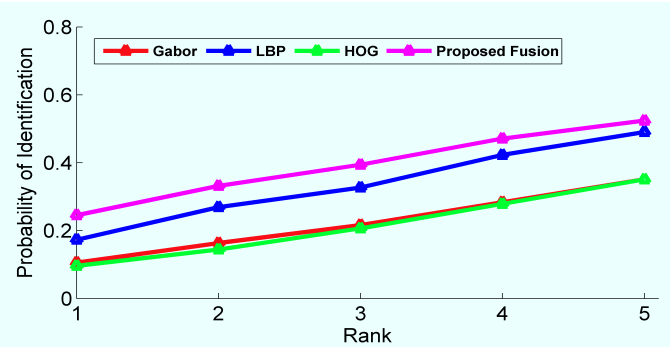

(d) Proposed With Mask

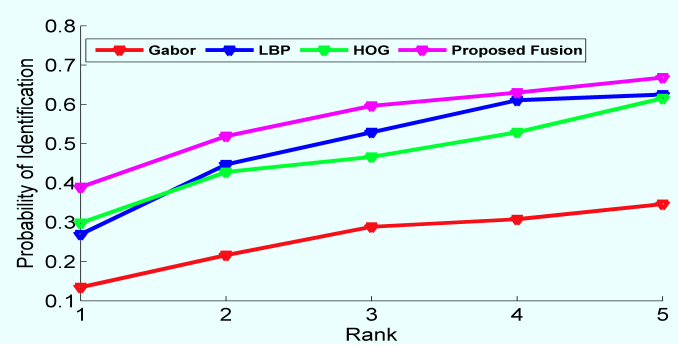

(f) Proposed Without Mask

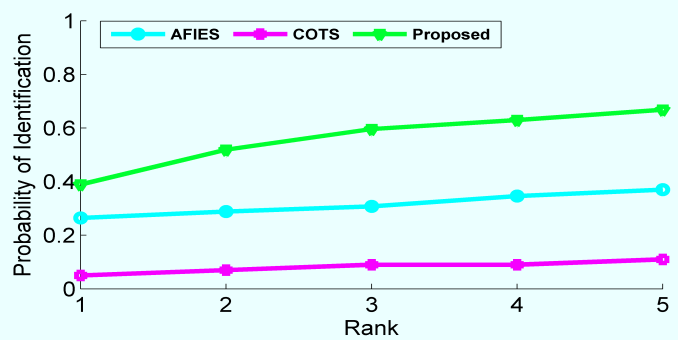

(h) AFIES and COTS vs. Proposed

Figure 9: Cross-spectral, cross-distance matching scenarios for VIS 1.5m (Gallery) against NIR $30 \mathrm{~m}$ (Probe) (Left) and VIS 1.5m (Gallery) against NIR $60 \mathrm{~m}$ (Probe) (Right). 
performance results compared to AFIES and COTS system, for gallery at $30 \mathrm{~m}$ and probe at $60 \mathrm{~m}$, the identification rank-1 accuracy is $56 \%$ (without mask) from proposed method, $19 \%$ from AFIES and $48 \%$ from COTS. Our proposed method achieves significant improvement over the academic and commercial method for $60 \mathrm{~m}$ and $90 \mathrm{~m}$ distance. For $60 \mathrm{~m}$, the identification accuracy is, $37 \%$ for proposed MFSDF, $22 \%$ for AFIES and 3\% from COTS. For 90m, the identification accuracy is, $44 \%$ for proposed MFSDF, $23 \%$ for AFIES and 2\% from COTS. For $120 m$, results are similar from proposed and AFIES (15\%) and very low performance results from COTS.

The CMC curves for first 5 ranks, for the best performing set out of randomly selected 4 different sets, are represented in Fig. 9. The identification accuracies are $58 \%$ for without mask, $52 \%$ for with mask from proposed system, $21 \%$ for AFIES and $51 \%$ for COTS.

COTS system provided us with good results only for one case, where we matched the gallery images against NIR $30 \mathrm{~m}$. The main reason for better performance of commercial system is that images are still with good quality. In our work, we collected the database under un-controlled conditions at long distances (for example at $60 \mathrm{~m}, 90 \mathrm{~m}$ and $120 \mathrm{~m}$ ). The poor quality of the images (at long distances) results in poor performance of the COTS system.

In order to examine the effectiveness of FR algorithms, each experiment is repeated several times (i.e. each time a different training set was randomly selected) for cross-scenarios using academic, commercial and proposed face recognition methods. To see the performance variation between three FR matchers, we first plotted the boxplots. In Fig. 10, where the first 2 figures are for cross distance, and rest of the 4 figures are for cross spectral face matching. 
In this we compared the rank-1 score from proposed method with AFIES. For COTS, it performed well only for VIS $1.5 \mathrm{~m} v \mathrm{~s}$. NIR $30 \mathrm{~m}$ and scores are very low for rest of the 7 sets when gallery data (NIR 30m, VIS $1.5 \mathrm{~m})$ is compared against probe data $(60 \mathrm{~m}, 90 \mathrm{~m}, 120 \mathrm{~m})$ as represented in Table 5 for cross distance and Table 6 for cross spectral experiments.

Based on the mean and variance of boxplots, the matching accuracy from proposed method (using either with or without mask), for NIR 30m vs. NIR 90m, is overall higher from proposed method compared to AFIES. For NIR $30 m$ vs. NIR $120 \mathrm{~m}$ and from cross-spectral (all the 4 cases c, d, e and f), our proposed method outperformed AFIES.

\subsection{Matching Results from Grouping in the Context of Demographic Information}

The use of demographic information (gender class) is proposed to improve the performance of face recognition systems. In our work, three different face recognition experiments are conducted: AFIES, COTS and proposed MFSDF method. For each FR cross-scenario, experiments are conducted, for example for crossdistance, NIR vs. NIR (30m to 60m, 90m and 120m) and for cross-spectral VIS vs. NIR (1.5m to $30 \mathrm{~m}, 60 \mathrm{~m}, 90 \mathrm{~m}$ and $120 \mathrm{~m})$. Each experiment is repeated several times for two FR cross-scenarios. For each demographic group (male and female), in total 63 FR experiment are conducted considering two FR cross-scenarios as represented in Table. 3.

Demographic-based Cross-Distance Matching: For cross-distance scenario, based on the results for male class, for $60 \mathrm{~m}$ and $90 \mathrm{~m}$ (probe images), we determined that identifications results are similar for proposed and AFIES method. From proposed method, the rank-1 score accuracy is $94 \%$ for $60 \mathrm{~m}$ and $81 \%$ for $90 \mathrm{~m}$ as shown in Table.7. For $120 \mathrm{~m}$ distance, our proposed method performs better and the rank-1 


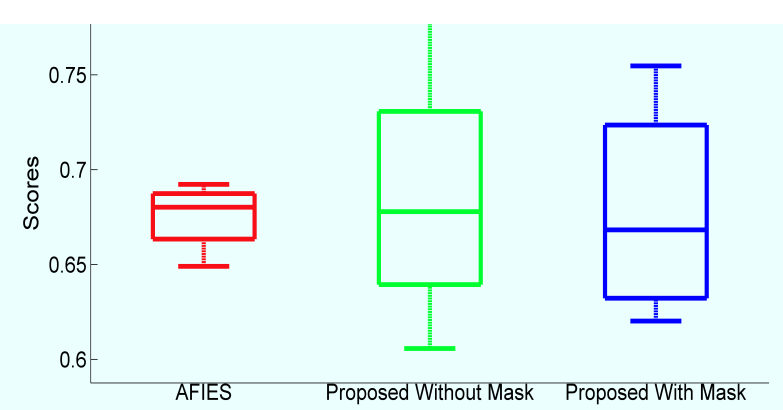

(a) NIR 30m vs. NIR 90m

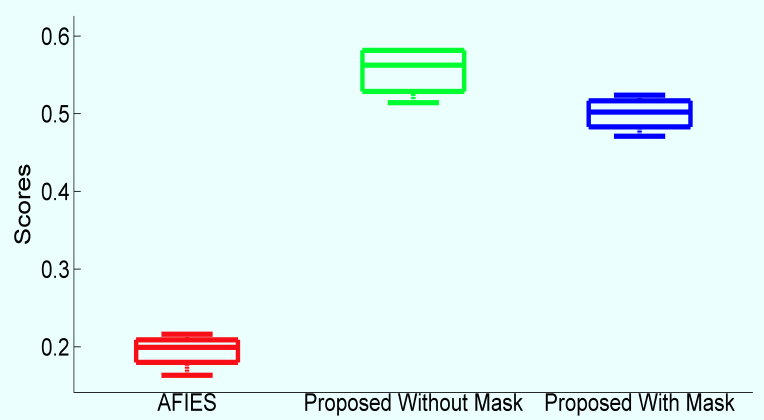

(c) VIS $1.5 \mathrm{~m}$ vs. NIR $30 \mathrm{~m}$

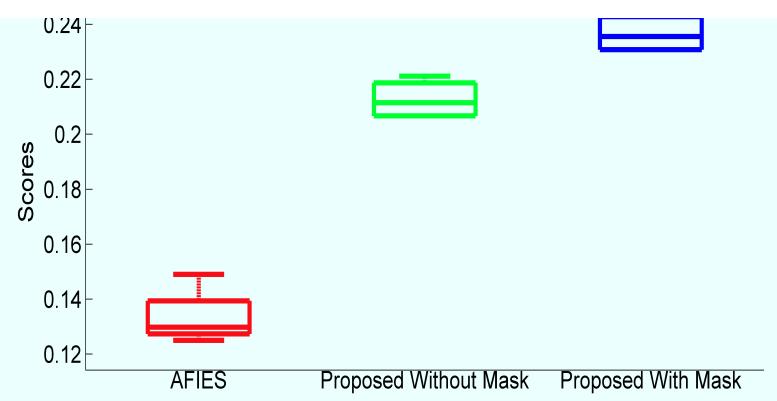

(e) VIS $1.5 \mathrm{~m}$ vs. NIR $90 \mathrm{~m}$

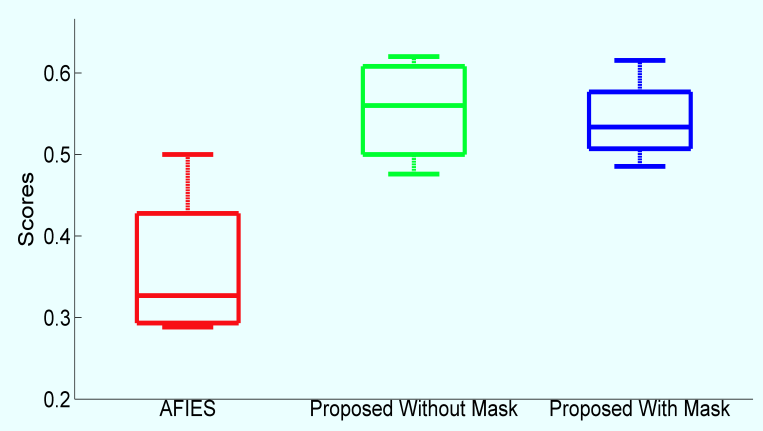

(b) NIR 30m vs. NIR $120 \mathrm{~m}$

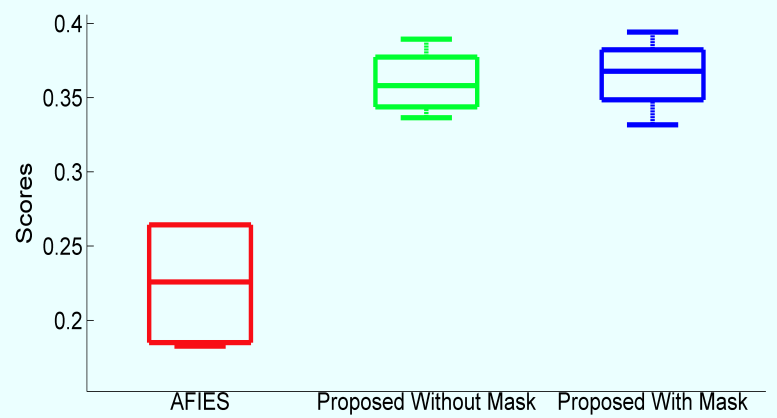

(d) VIS $1.5 \mathrm{~m}$ vs. NIR $60 \mathrm{~m}$

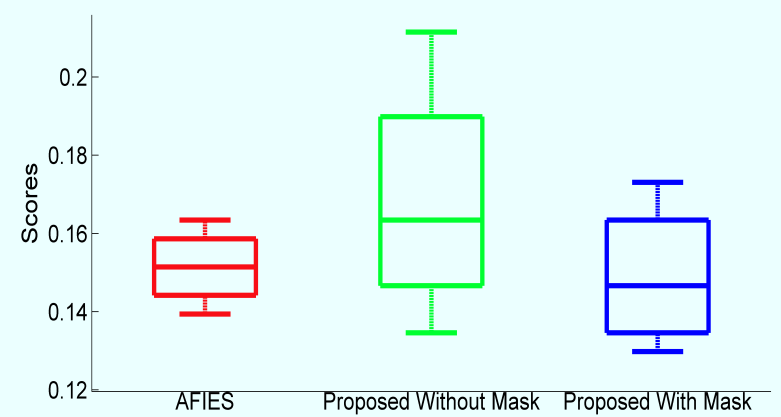

(f) VIS $1.5 \mathrm{~m}$ vs. NIR $120 \mathrm{~m}$

Figure 10: Box-plots for cross-distance using intra-spectral and cross-spectral matching scenarios after running each experiment several times. 
Table 7: In this table we investigate the intra-spectral, cross-distance matching scenarios for database after the usage of the context of demographic information: experimental results when running all FR algorithms using 50\% of the NIR data for training and rest of the data for testing. For each algorithm including, AFIES, COTS and proposed MFSDF, the experiments were run several times and the rank-1 scores presented here are the means.

\begin{tabular}{|c|c|c|c|}
\hline \multirow[b]{2}{*}{ FR Algorithm } & \multicolumn{3}{|c|}{ Intra-Spectral (NIR vs. NIR), Cross-Distance Matching (Rank-1) } \\
\hline & $\begin{array}{c}30 \text { vs. } 60 \mathrm{~m} \\
50 \%\end{array}$ & $\begin{array}{c}30 \text { vs. } 90 \mathrm{~m} \\
50 \%\end{array}$ & $\begin{array}{l}30 \text { vs. } 120 \mathrm{~m} \\
50 \%\end{array}$ \\
\hline \multicolumn{4}{|l|}{ MALE DATA } \\
\hline \multicolumn{4}{|l|}{ AFIES } \\
\hline Bayesian MAP & 0.92 & 0.69 & 0.40 \\
\hline Bayesian ML & 0.93 & 0.74 & 0.47 \\
\hline LDA Euclidean & 0.93 & 0.69 & 0.45 \\
\hline LDA lda_Soft & 0.93 & 0.69 & 0.47 \\
\hline PCA Euclidean & 0.79 & 0.39 & 0.28 \\
\hline PCA MahaCosine & 0.94 & 0.79 & 0.52 \\
\hline \multicolumn{4}{|l|}{ Proposed Methods } \\
\hline MFSDF Without Mask & 0.92 & 0.78 & 0.57 \\
\hline MFSDF With Mask & 0.92 & 0.71 & 0.52 \\
\hline \multicolumn{4}{|l|}{ Fusion of MFSDF (best set) } \\
\hline With and Without Mask & 0.94 & 0.81 & 0.71 \\
\hline \multicolumn{4}{|l|}{ FEMALE DATA } \\
\hline \multicolumn{4}{|l|}{ AFIES } \\
\hline Bayesian MAP & 0.78 & 0.53 & 0.38 \\
\hline Bayesian ML & 0.82 & 0.56 & 0.42 \\
\hline LDA Euclidean & 0.74 & 0.47 & 0.38 \\
\hline LDA lda_Soft & 0.75 & 0.46 & 0.39 \\
\hline PCA Euclidean & 0.56 & 0.21 & 0.17 \\
\hline PCA MahaCosine & 0.84 & 0.61 & 0.49 \\
\hline \multicolumn{4}{|l|}{ Proposed Methods } \\
\hline MFSDF Without Mask & 0.65 & 0.51 & 0.44 \\
\hline MFSDF With Mask & 0.71 & 0.47 & 0.41 \\
\hline \multicolumn{4}{|l|}{ Fusion of MFSDF (best set) } \\
\hline With and Without Mask & 0.82 & 0.74 & 0.54 \\
\hline
\end{tabular}


score accuracy is improved from $52 \%$ to $71 \%$.

Based on the results for female class, for $60 \mathrm{~m}$ (probe images), we determined that identifications results are similar for proposed method and AFIES. From proposed method, the rank-1 score accuracy is $82 \%$ for $60 \mathrm{~m}$ as represented in Table.7. For $90 \mathrm{~m}$ and $120 \mathrm{~m}$, our proposed method performs better and the rank-1 score accuracy is improved from $61 \%$ to $74 \%$ for $90 \mathrm{~m}$ and $49 \%$ to $54 \%$ for $120 \mathrm{~m}$.

Demographic-based Cross-Spectral Matching: For cross-spectral scenario, based on the results for male class, we determined that proposed system outperformed both the commercial and academic software for all four cases including, when gallery images (VIS $1.5 \mathrm{~m}$ ) matched against probe images (NIR 30m, NIR 60m, NIR 90m and NIR 120m). For 30m, identification performance is improved from $34 \%$ (AFIES), $51 \%$ (COTS) to $61 \%$ (Proposed). For $60 m$, identification performance is improved from 33\% (AFIES), 3\% (COTS) to $46 \%$ (Proposed) and $26 \%$ (AFIES), $2 \%$ (COTS) to $29 \%$ (Proposed) for $90 \mathrm{~m}$. The rank-1 score accuracy for $120 \mathrm{~m}$ is improved from $18 \%$ (AFIES) to $30 \%$ (Proposed).

Based on the results for female class, for NIR 30m, COTS system performs better than both academic and proposed system. The demographic information and good quality of the images is the main reason for better performance of commercial system. The results for (AFIES) and proposed method are similar in performance. Moreover, for each of these three FR matchers (AFIES, COTS and proposed MFSDF), performance of the system is not improved and rank-1 scores are close to results without any grouping in context of demographic information. In some of the cross scenarios, FR system did not perform well and the rank-1 score is decreased (after grouping of the data into female class) from $23 \%$ (without grouping in the context of demographic information) to $18 \%$ (AFIES) for NIR 
Table 8: In this table we investigate the cross-spectral, cross-distance matching scenarios for database after the usage of grouping in the context of demographic information: experimental results when running using 50\% of the data for training and the rest data for testing. Experiments were run several times and the rank-1 scores presented here are the means.

\begin{tabular}{|c|c|c|c|c|}
\hline \multirow{3}{*}{$\begin{array}{l}\text { VIS } 1.5 m \text { vs. } \\
\text { FR Algorithm }\end{array}$} & \multicolumn{4}{|c|}{ Cross-Spectral (VIS vs. NIR), Cross-Distance Matching (Rank-1) } \\
\hline & NIR $30 \mathrm{~m}$ & NIR $60 \mathrm{~m}$ & NIR $90 \mathrm{~m}$ & NIR $120 \mathrm{~m}$ \\
\hline & $\mathbf{5 0 \%}$ & $\mathbf{5 0 \%}$ & $\mathbf{5 0 \%}$ & $\mathbf{5 0 \%}$ \\
\hline \multicolumn{5}{|l|}{ MALE DATA } \\
\hline \multicolumn{5}{|l|}{ AFIES } \\
\hline Bayesian MAP & 0.34 & 0.33 & 0.18 & 0.14 \\
\hline Bayesian ML & 0.31 & 0.29 & 0.19 & 0.14 \\
\hline LDA Euclidean & 0.30 & 0.27 & 0.25 & 0.17 \\
\hline LDA lda_Soft & 0.32 & 0.28 & 0.26 & 0.18 \\
\hline PCA Euclidean & 0.17 & 0.15 & 0.15 & 0.08 \\
\hline PCA MahaCosine & 0.20 & 0.26 & 0.18 & 0.14 \\
\hline COTS & 0.52 & 0.03 & 0.02 & 0.02 \\
\hline \multicolumn{5}{|l|}{ Proposed Methods } \\
\hline MFSDF Without Mask & 0.47 & 0.39 & 0.24 & 0.23 \\
\hline MFSDF With Mask & 0.51 & 0.39 & 0.23 & 0.25 \\
\hline \multicolumn{5}{|l|}{ Fusion of MFSDF (best set) } \\
\hline With and Without Mask & 0.61 & 0.46 & 0.29 & 0.30 \\
\hline \multicolumn{5}{|l|}{ FEMALE DATA } \\
\hline \multicolumn{5}{|l|}{ AFIES } \\
\hline Bayesian MAP & 0.30 & 0.25 & 0.17 & 0.12 \\
\hline Bayesian ML & 0.31 & 0.22 & 0.17 & 0.12 \\
\hline LDA Euclidean & 0.26 & 0.21 & 0.18 & 0.14 \\
\hline LDA lda_Soft & 0.27 & 0.23 & 0.18 & 0.12 \\
\hline PCA Euclidean & 0.20 & 0.13 & 0.11 & 0.11 \\
\hline PCA MahaCosine & 0.19 & 0.16 & 0.12 & 0.23 \\
\hline COTS & 0.65 & 0.08 & 0.08 & 0.03 \\
\hline \multicolumn{5}{|l|}{ Proposed Methods } \\
\hline MFSDF Without Mask & 0.26 & 0.22 & 0.18 & 0.19 \\
\hline MFSDF With Mask & 0.32 & 0.27 & 0.26 & 0.16 \\
\hline
\end{tabular}




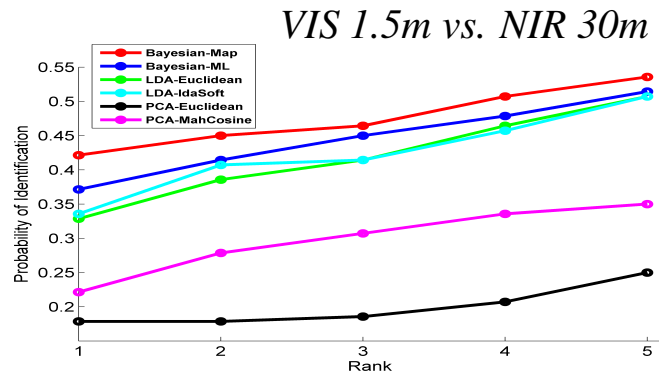

(a) AFIES (VIS vs. NIR 30m)

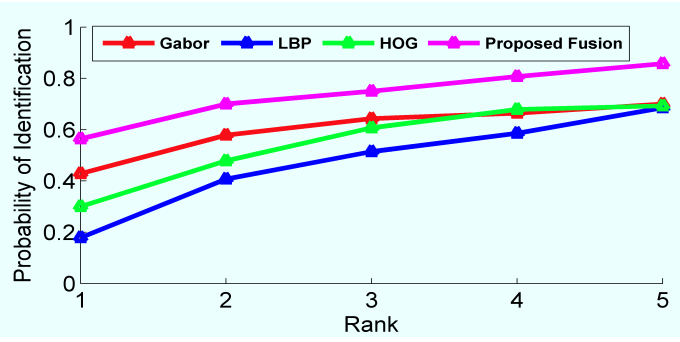

(c) Proposed With Mask

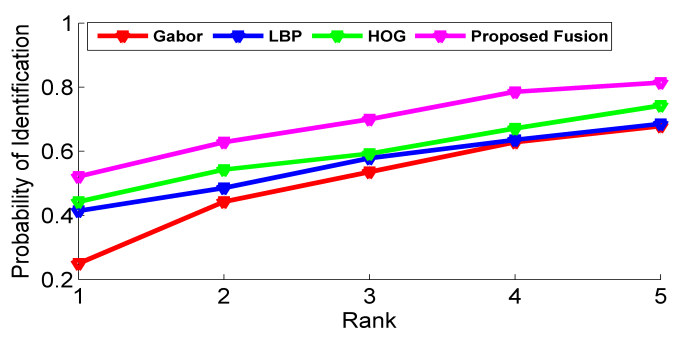

(e) Proposed Without Mask

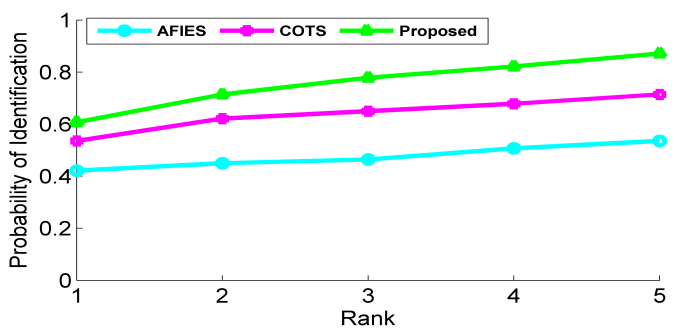

(g) AFIES and COTS vs. Proposed

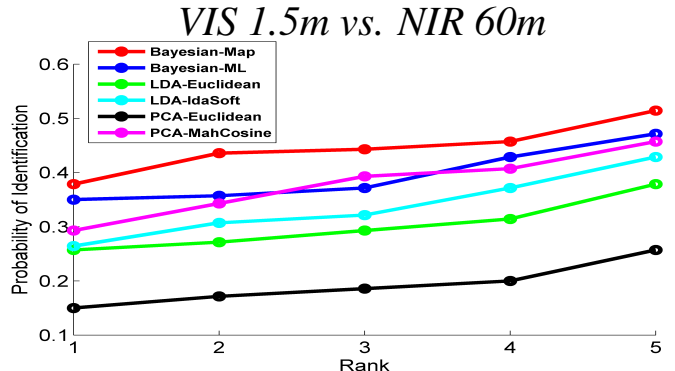

(b) AFIES (VIS vs. NIR 60m)

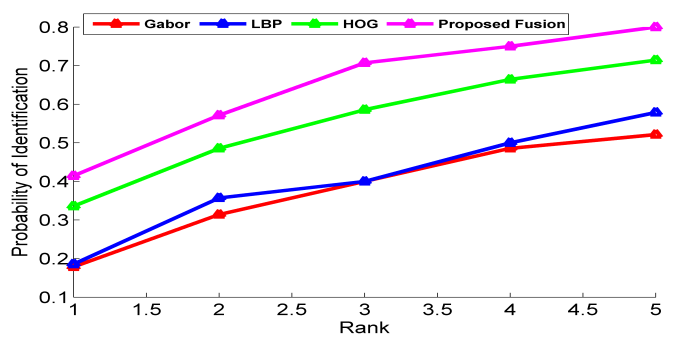

(d) Proposed With Mask

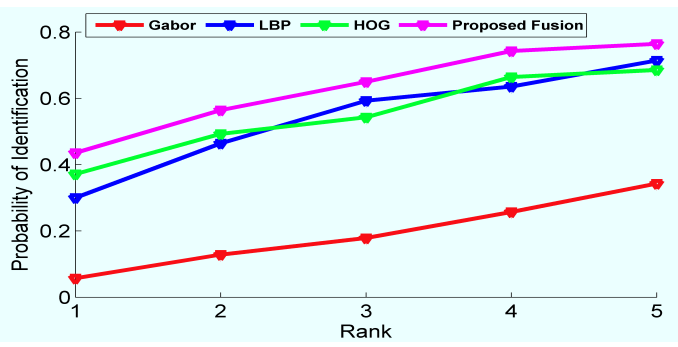

(f) Proposed Without Mask

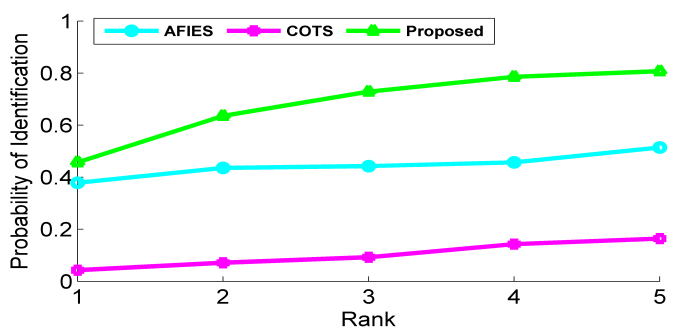

(h) AFIES and COTS vs. Proposed

Figure 11: Cross-spectral matching scenarios for Male Data, VIS 1.5m (Gallery) against NIR $30 \mathrm{~m}$ (Probe) (Left) and VIS 1.5m (Gallery) against NIR $60 \mathrm{~m}$ (Probe) (Right). 
$90 m, 56 \%$ (without demographic information) to $32 \%$ (proposed) for NIR $30 \mathrm{~m}$ and $37 \%$ to $27 \%$ (proposed) for NIR $60 m$ of the system.

Based on the results, we conclude that (for all three FR matchers), female class is more challenging to match in comparison to male class (with grouping in the context of demographic information) and all data (without grouping). Klare et al. [47] reported that, factors such as, use of cosmetics and small size of female face, results in higher within-class variance and affects the identification performance of system and supported our findings.

\subsection{Face Matching With Extended Gallery Data}

In this we used Long Distance Heterogeneous Face Database (LDHF) database collected from Kang et al. [8], with 100 subjects to extend the gallery data. LDHF data contains VIS and NIR images at distance of $1 \mathrm{~m}, 60 \mathrm{~m}, 100 \mathrm{~m}$ and $150 \mathrm{~m}$. To perform cross-distance face matching experiments, NIR images collected at distance from $1 \mathrm{~m}$ to $150 \mathrm{~m}$ are selected. For cross-spectral, VIS images at a distance of $1 \mathrm{~m}$ and NIR images at a distance of $1 \mathrm{~m}, 60 \mathrm{~m}, 100 \mathrm{~m}$ and $150 \mathrm{~m}$ distance are selected. We repeated the same set of experiments, performed with and without the usage of the context of demographic information using cross-distance and cross-spectral face matching scenarios.

For cross-distance face matching, results are presented in Table 9. Based on the results, we concluded that for $60 \mathrm{~m}$, identification rank-1 performance is higher for AFIES system compared to our proposed system for with and without the usage of grouping in the context of demographic information. For $90 \mathrm{~m}$ and $120 \mathrm{~m}$, our proposed system achieves better performance results than AFIES. For example, the rank-1 score accuracies at $90 \mathrm{~m}$ and $120 \mathrm{~m}$ distances are $42 \%$ and $30 \%$ (All Data), $48 \%$ and 36\% (Male Class) and 25\% and 20\% (Female Class) respectively. 
Table 9: In this table we investigate the intra-spectral, cross-distance matching scenarios with and without grouping in the context of demographic information, with extended gallery: experimental results when running all FR algorithms using LDHF data for training and our data for testing.

\begin{tabular}{|c|c|c|c|}
\hline \multirow[b]{2}{*}{ FR Algorithm } & \multicolumn{3}{|c|}{ Intra-Spectral (NIR vs. NIR), Cross-Distance Matching (Rank-1) } \\
\hline & $\begin{array}{c}30 \text { vs. } 60 \mathrm{~m} \\
50 \%\end{array}$ & $\begin{array}{c}30 \text { vs. } 90 \mathrm{~m} \\
50 \%\end{array}$ & $\begin{array}{c}30 \text { vs. } 120 \mathrm{~m} \\
50 \%\end{array}$ \\
\hline \multicolumn{4}{|l|}{ ALL DATA } \\
\hline \multicolumn{4}{|l|}{ AFIES } \\
\hline Bayesian MAP & 0.77 & 0.34 & 0.12 \\
\hline Bayesian ML & 0.78 & 0.38 & 0.20 \\
\hline LDA Euclidean & 0.69 & 0.35 & 0.20 \\
\hline LDA lda_Soft & 0.36 & 0.11 & 0.07 \\
\hline PCA Euclidean & 0.58 & 0.21 & 0.09 \\
\hline PCA MahaCosine & 0.67 & 0.31 & 0.17 \\
\hline Proposed Methods & & & \\
\hline MFSDF Without Mask & 0.71 & 0.42 & 0.30 \\
\hline \multicolumn{4}{|l|}{ MALE DATA } \\
\hline \multicolumn{4}{|l|}{ AFIES } \\
\hline Bayesian MAP & 0.84 & 0.43 & 0.30 \\
\hline Bayesian ML & 0.84 & 0.42 & 0.30 \\
\hline LDA Euclidean & 0.75 & 0.39 & 0.15 \\
\hline LDA lda_Soft & 0.37 & 0.15 & 0.05 \\
\hline PCA Euclidean & 0.65 & 0.23 & 0.11 \\
\hline PCA MahaCosine & 0.73 & 0.32 & 0.18 \\
\hline Proposed Methods & & & \\
\hline MFSDF Without Mask & 0.80 & 0.48 & 0.36 \\
\hline \multicolumn{4}{|l|}{ FEMALE DATA } \\
\hline \multicolumn{4}{|l|}{ AFIES } \\
\hline LDA Euclidean & 0.44 & 0.11 & 0.17 \\
\hline LDA lda_Soft & 0.11 & 0.05 & 0.09 \\
\hline PCA Euclidean & 0.41 & 0.13 & 0.15 \\
\hline PCA MahaCosine & 0.46 & 0.13 & 0.07 \\
\hline Proposed Methods & & & \\
\hline MFSDF Without Mask & 0.45 & 0.25 & 0.20 \\
\hline
\end{tabular}




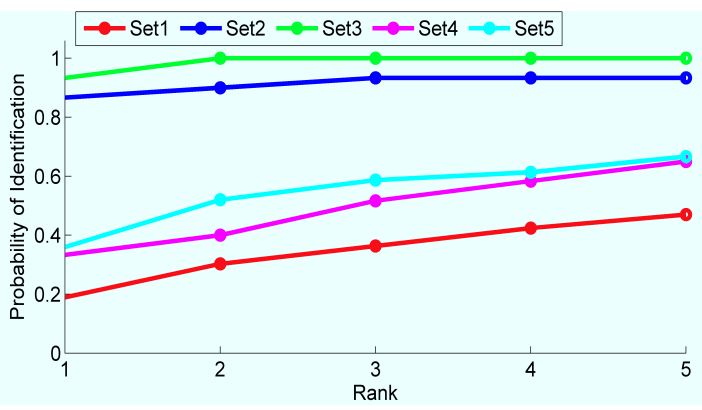

(a) Female: VIS $1.5 \mathrm{~m}$ vs. NIR $30 \mathrm{~m}$

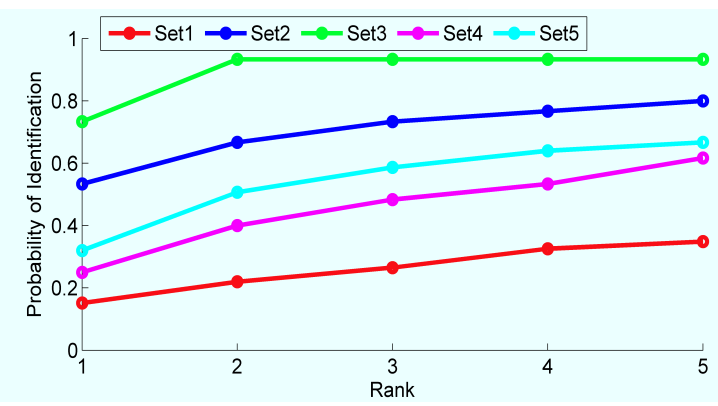

(c) Female: VIS $1.5 \mathrm{~m}$ vs. NIR $60 \mathrm{~m}$

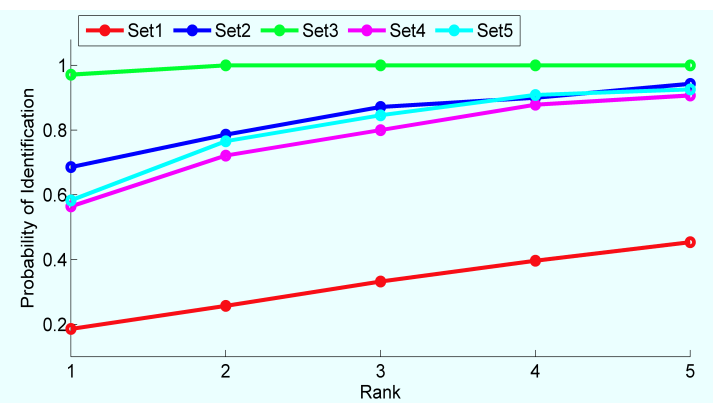

(b) Male: VIS $1.5 \mathrm{~m}$ vs. NIR 30m

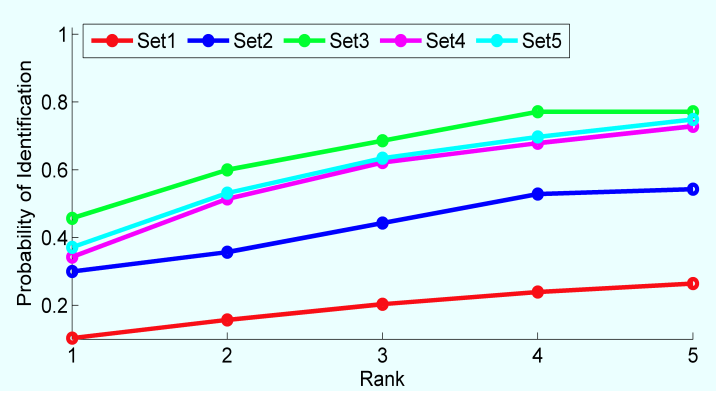

(d) Male: VIS $1.5 \mathrm{~m}$ vs. NIR $60 \mathrm{~m}$

Figure 12: Cross-spectral matching scenarios for VIS $1.5 \mathrm{~m}$ against NIR 30m (top) and VIS $1.5 m$ against NIR $60 m$ (bottom) with extended gallery set (with grouping in context of demographic information).

493

For cross-spectral scenario, we conducted face matching experiments using 5 different sets. For Set1, LDHF data base is used for training and our database for testing. Set2, where our database is used for training and LDHF database for testing. Set 3 to Set 5 are based on selection of both the databases to train the system (our and LDHF data) and rest of the data for testing. For example for Set3 LDHF data is used for testing, in Set4 our data is used for testing and, finally, in Set5 both are used for testing (non-overlapping of training/testing). 
Table 10: Measure of statistics (mean, median, minimum and maximum) for attained image quality scores: experimental results when visible images are used as reference images and long distance images (NIR 60m) as query images.

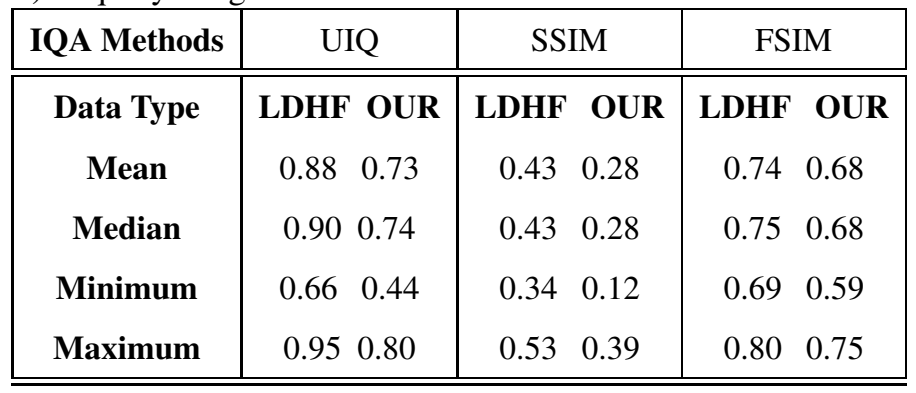

Based on the results, we conclude that the best performance results are achieved from Set3, where both LDHF and our data is used for training and only LDHF database is used for testing. The main reason for better performance for Set3 is the quality of the images. To verify our face recognition results, a number of image quality assessment (IQA) methods are used. Since image quality indicators have been used in FR experiments before, providing valuable information, we decided to use them in this paper. For example, universal image quality $(U I Q)$ was used in FR experiments [48, 49], structural similarity index method (SSIM) [50] and feature similarity index for image quality (FSIM). The visible images collected under controlled conditions (VIS 1.5m) are used as reference images. The images collected at a distance of $60 \mathrm{~m}$ in NIR band are selected as query images. The quality score values are lies between 0 and 1 .

Experimental results showed that for LDHF database (100 subjects), quality scores mean value is 0.88 for UIQ, 0.43 for SSIM and 0.74 for FSIM. On the other hand, for our database (103 subjects), quality scores mean value is 0.73 for UIQ, 0.28 for SSIM and 0.68 for FSIM. The statistical analysis results for quality scores (for both the databases) are presented in Table 10. 


\section{Discussion and Conclusions}

The night-time mid-range NIR dataset represents a very challenging realworld FR scenario. We conducted a comprehensive data collection with a sensor capable of acquiring NIR images at standoff distances ranging from 30-120m. The data collection effort (face images of subjects were acquired with intra-personal variability) was designed to investigate the hypothesis that night time mid-range NIR imagery would yield high recognition performance at short ranges that degrades as the distance of the target to the sensor increases. Different scenarios were tested, allowing us to gain some understanding of the shortcomings of such a FR scenario. As expected, intra-spectral (NIR) intra-distance experiments resulted in a performance that reduces as a function of distance, especially at ranges greater than 90 meters, where facial features become less prominent due to various factors including atmospheric conditions. Generally, all rank-1 rates appear high (some matchers perform much better than others, e.g. LDA and Bayesian MAP vs. PCA), but this can be explained by the fact that we were matching same session data. When cross-distance matching experiments were performed and the gallery set is composed of either NIR or visible face images, we determined that the closer the standoff distance we operate to acquire face images at night the better the identification performance is. In addition, the higher the training set size, better is the identification performance.

We performed the identification experiments for three types of cross-scenarios: (i) intra-distance and intra-spectral, (ii) cross-distance and intra-spectral, and (iii) cross-spectral and cross-distance. We proposed a multi-feature scenario dependent fusion face matching scheme and evaluated the performance improvement by comparing with available academic and commercial systems. Our results provide 
important evidence towards whether the proposed system can be taken into reality. The main requirements for a real time face recognition system are: it should be able to do the recognition for the database collected in challenging environments, and, less time consuming for example can match the query data within seconds.

First, it is clear that our proposed method (MFSDF) can enhance the automated FR performance for the cross-scenarios. In the experimental results, it was shown that the proposed system provides better performance results than both AFIES and COTS systems, for cross distance (particularly for 30 vs. $90 \mathrm{~m}$ and $120 \mathrm{~m}$ ) which initially resulted in the lowest accuracy using the COTS system. For cross spectral scenarios, proposed system achieves better rank-1 score accuracy, particularly for VIS $1.5 \mathrm{~m}$ vs. $30 \mathrm{~m}$, where the lowest accuracy recorded from the AFIES system and for VIS $1.5 \mathrm{~m}$ vs. NIR $60 \mathrm{~m}$ the lowest accuracy recorded from the COTS system. This suggests that our proposed method increases the FR performance of our biometric system.

Second, through our experiments using grouping in the context of demographic information of the data, where we filtered the data based on gender class (male and female), it was shown that male class provides improvement in the results for cross-distance and cross-spectral scenarios. On the other hand, female class is more challenging to perform the face matching for all the matchers used in this work as expected. We have a valid reason for the poor performance using female class, for example the factors like facial makeup, size of face etc. With extended gallery with more variation in the training data (including data collected under different conditions like sensors, distances), where LDHF data is used for training and our data for testing and vice versa, both the databases are used for training and testing, it was shown that, it will improve the robustness of the face 
recognition method as expected.

Third, We proposed a system that is efficient in comparison to the available traditional methods. The time elapsed to run the cross-scenario experiments including the training and testing part, is few seconds (e.g. 3 to 4 seconds). When compared to our proposed system, the traditional FR systems used and tested were much slower - it took hours to run the same set of experiments. At present, the programming platform used to develop the proposed system is Matlab. In the future we plan to use $\mathrm{C}++$ so that we can develop a capability for real-time processing.

Beyond the concrete results and methods that were presented, there exist many fruitful avenues for future extensions. For example, one could experiment with more demographic filters like age, ethnicity etc. Most importantly, though, one could investigate an important practical consideration: in our experiments, we have performed this grouping in the context of demographic information manually. However, in autonomous practical systems, these have to be obtained automatically. Obtaining demographic information automatically is thus a fruitful area for future research towards wider applicability of automated face recognition systems that can perform well under difficult conditions.

\section{Acknowledgment}

This material is sponsored partly through the start-up funds of Dr. Bourlai, and is also based upon work supported by the Center for Identification Technology Research and the National Science Foundation under Grant No. - West Virginia University 1066197. The authors are also grateful to Dr. J. Dawson, Nathan Kalka and other WVU faculty and students that supported this work. Thanks to Chuck Coleman (Supervisor Sr. Lab Instr. Spec., Mechanical and Aerospace 
Engineering, WVU) for designing and developing the weatherproof enclosure of our camera system. Thanks to J. VonDollen for his help to design the experimental set up and to collect the data as part of his Masters degree. Finally, special thanks to all Vumii Imaging Inc. personnel for their great support and guidance to help us prepare the system to its current operational form.

\section{References}

[1] M. Pietikinen, Image analysis with local binary patterns, in: Proc. Scandinavian Conf. Image Analysis (IA), 2005, pp. 115-118.

[2] P. Belhumeur, J. Hespanha, D. J. Kriegman, Eigenfaces vs. fisherfaces: Recognition using class specific linear projection, IEEE Trans. Pattern Anal. Mach. Intell. 19 (1997) 711-720.

[3] T. Bourlai, Mid-wave ir face recognition systems, in: SPIE Newsroom Magazine - Defense \& Security, 2013, pp. 1-3.

[4] T. Bourlai, Short-wave infrared for face-based recognition systems, in: SPIE Newsroom Magazine - Defense \& Security, 2012, pp. 1-2.

[5] W. Zhao, R. Chellappa, A. Rosenfeld, P. Phillips, Face recognition: A literature survey, ACM Computing Surveys (2006) 399-458.

[6] Y. Yao, B. R. Abidi, R. Besma, N. D. Kalka, N. A. Schmid, M. A. Abidi, A. Mongi., Improving long range and high magnification face recognition: Database acquisition, evaluation, and enhancement, Computer Vision and Image Understanding 111. 
[7] T. Bourlai, J. V. Dollen, N. Mavridis, C. Kolanko, Evaluating the efficiency of a nighttime, middle-range infrared sensor for applications in human detection and recognition, in: Proc. SPIE Infrared Imaging Systems: Design, Analysis, Modeling, and Testing XXIII, Bellingham, WA, USA, 2012, pp. $1-12$.

[8] D. Kang, H. Han, A. K. Jain, S.-W. Lee, Nighttime face recognition at large standoff: Cross-distance and cross-spectral matching, Pattern Recognition 47 (12) (2014) 3750-3766.

[9] J. C. Klontz, A. K. Jain, A case study on unconstrained facial recognition using the boston marathon bombings suspects, in: Technical Report MSUCSE-13-4, 2013, pp. 1-8.

[10] N. Kalka, T. Bourlai, B. Cukic, L. Hornak, Cross-spectral Face recognition in Heterogeneous Environments: A Case Study on Matching Visible to Short-wave Infrared Imagery, in: International Joint Conference on Biometrics, 2011, pp. 1-8.

[11] A. Reza, Realization of contrast limited adaptive histogram equalization (clahe) for real-time image enhancement, VLSI Signal Processing 38 (2004) $35-44$.

[12] M. Turk, A. Pentland, Face recognition using eigenfaces, in: IEEE Conference on Computer Vision and Pattern Recognition (CVPR), 1991, pp. $586-591$.

[13] Y. D. Zhang, A. Frangi, J. Yang, Two-dimensional pca: a new approach to 
appearance based face representation and recognition, IEEE Transactions on Pattern Analysis and Machine Intelligence (PAMI) 26 (2004) 131-137.

[14] L. Wiskott, J.-M. Fellous, N. Kruger, C. von der Malsburg, Face recognition by elastic bunch graph matching, in: Proc. IEEE International Conference on Image Processing (ICIP), 1997, pp. 129-132.

[15] H. Yu, J. Yang, A direct lda algorithm for high-dimensional data with application to face recognition, IEEE Transactions on Pattern Recognition 34 (2001) 2067-2070.

[16] A. Nefian, M. Hayes, Face detection and recognition using hidden markov models, in: Proc. IEEE Conference on Image Processing (ICIP), 1998, pp. $141-145$.

[17] W. A. Yang, A. Ganesh, S. Sastry, Y. Ma, Robust face recognition via sparse representation, IEEE Transactions on Pattern Analysis and Machine Intelligence (PAMI) 31 (2009) 210-227.

[18] Y. Su, S. Shan, X. Chen, W. Gao, Hierarchical ensemble of global and local classifiers for face recognition, IEEE Transactions on on Image Processing 18 (2009) 1885-1896.

[19] Facevacs software developer kit, cognitec systems gmbh, http://www.cognitec-systems.de.

[20] I. Ltkebohle, Pittpatt software developer kit, pittsburgh pattern recognition, inc., http://www.pittpatt.com.

[21] L1 systems (identity tools g8, http:// www.11id.com. 
[22] H. Maeng, S. Liao, D. Kang, S.-W. Lee, , A. K. Jain, Nighttime face recognition at long distance: Cross-distance and cross-spectral matching, in: Asian Conference on Computer Vision Conference on Computer Vision (ACCV), 2012, pp. 5-9.

[23] T. Bourlai, N. Narang, B. Cukic, L. Hornak, On designing a Swir MultiWavelength Facial-based acquisition system, in: Proc. SPIE, Vol. 8353, 2012, pp. 83530R-83530R-14.

[24] X. Tan, B. Triggs, Enhanced local texture feature sets for face recognition under difficult lighting conditions, Image Processing, IEEE Transactions on 19 (6) (2010) 1635-1650.

[25] T. Bourlai, B. Cukic, Multi-spectral face recognition: Identification of people in difficult environments, in: Proc. IEEE Conference on Intelligence and Security Informatics (ISI), Washington DC, USA, 2012, pp. 196 - 201.

[26] D. Huang, Y. W. andY. Wang, A robust method for near infrared face recognition based on extended local binary pattern, in: in Proc. ISVC, 2007, pp. 437-446.

[27] Z. Pan, G. E. Healey, M. Prasad, B. J. Tromberg, Face recognition in hyperspectral images, IEEE Trans. Pattern Anal. Mach. Intell. 25 (2003) 15521560 .

[28] B. Zhang, L. Zhang, D. Zhang, L. Shen, Directional binary code with application to polyu near-infrared face database, Pattern Recogn. Lett. 31 (2010) 2337-2344. 
[29] S. Z. Li, R. Chu, S. Liao, L. Zhang, Illumination invariant face recognition using near-infrared images, IEEE Trans. Pattern Anal. Mach. Intell. 29 (2007) 627-639.

[30] S. Zhao, R.-R. Grigat, An automatic face recognition system in the near infrared spectrum, in: in Proc. MLDM, 2005, pp. 437-444.

[31] L. Shen, J. He, S. Wu, S. Zheng, Face recognition from visible and nearinfrared images using boosted directional binary code, in: in Proc. ICIC, 2012, pp. 404-411.

[32] J. Chen, D. Yi, J. Yang, G. Zaho, S. Z. Li, M. Pietikainen, Learning mappings for face synthesis from near infrared to visual light images, in: in Proc. IEEE CVPR, 2009, pp. 156-163.

[33] R. Wang, J. Yang, D. Yi, S. Z. Li, An analysis-by-synthesis method for heterogeneous face biometrics, in: in Proc. ICB, 2009, pp. 319-326.

[34] Z. Zhang, Y. Wang, Z. Zhang, Face synthesis from near-infrared to visual light via sparse representation, in: in Proc. IJCB, 2011, pp. 1-6.

[35] M. Davis, M. Smith, J. Canny, N. Good, S. King, R. J. R., Towards Contextaware Face Recognition, in: Proc. 13th ACM Conference on Multimedia, 2005, pp. 483-486.

[36] M. Davis, M. Smith, F. Stentiford, A. Bamidele, J. Canny, N. Good, S. King, R. Janakiraman, Using context and similarity for face and location identication, in: Proc. SPIE, 2006, pp. 1-10. 
[37] D. Lin, A. Kapoor, G. Hua, S. Baker, Joint people, event, and location recognition in personal photo collections using cross-domain context, in: Proc. ECCV, 2010, pp. 243-256.

[38] D. Yi, R. Liu, R. Chu, Z. Lei, S. Z. Li, Face matching between near infrared and visible light images, in: in Proc. ICB, 2007, pp. 523-530.

[39] S. Liao, D. Yi, Z. Lei, R. Qin, S. Z. Li, Heterogeneous face recognition from local structures of normalized appearance, in: in Proc. ICB, 2009, pp. 209-218.

[40] B. F. Klare, A. K. Jain, Heterogeneous face recognition using kernel prototype similarities, Pattern Analysis and Machine Intelligence, IEEE Transactions on 35 (6) (2013) 1410-1422.

[41] D. S. Bolme, J. R. Beveridge, M. L. Teixeira, B. A. Draper, The CSU face identification evaluation system: Its purpose, features and structure, in: Proc. International Conference on Vision Systems, Graz, Austria, 2003, pp. 304 311.

[42] L. Sirovich, M. Kirby, Application of the Karhunen-Loeve procedure for the characterization of human faces, Transactions on Pattern Analysis and Machine Intelligence 12 (1) (1990) 103-108.

[43] M. Turk, A. Pentland, Eigenfaces for recognition, Journal of Cognitive Neuroscience 3 (1) (1991) 71-86.

[44] A. P. Devijver, J. Kittler, Pattern recognition: A statistical approach, Prentice-Hall, Englewood Cliffs, N. J., 1982. 
[45] P. Belhumeur, J. Hespanha, D. J. Kriegman, Eigenfaces vs. fisherfaces: Recognition using class specific linear projection, Transactions on Pattern Analysis and Machine Intelligence 19 (1996) 45-58.

[46] M. Teixeira, The bayesian intrapersonal/extrapersonal classifier, Master's thesis, Colorado State University, Fort Collins, Colorado 80523 U.S.A. (2003).

[47] B. Klare, M. Burge, J. Klontz, R. V. Bruegge, A. K. Jain, Face recognition performance: Role of demographic information, Information Forensics and Security, IEEE Transactions on 7 (6) (2012) 1789-1801.

[48] T. Bourlai, A. Clark, L. Best-Rowden, Methodological insights on restoring face photos of multinational passports, in: Technologies for Homeland Security (HST), 2013 IEEE International Conference on, 2013, pp. 54-60.

[49] A. Abaza, M. A. Harrison, T. Bourlai, Quality metrics for practical face recognition, in: Pattern Recognition (ICPR), 2012 21st International Conference on, 2012, pp. 3103-3107.

[50] Y. Sun, M. Tistarelli, D. Maltoni, Structural similarity based image quality map for face recognition across plastic surgery, in: Biometrics: Theory, Applications and Systems (BTAS), 2013 IEEE Sixth International Conference on, 2013, pp. 1-8. 
and with mask (Fig. 5) based on normalization technique developed from AFIES (more detail information is provided in the section 3.3 on pages 13-14). 